\title{
A Multibaseline Pol-InSAR Inversion Scheme for Crop Parameter Estimation at Different Frequencies
}

\author{
Manuele Pichierri, Irena Hajnsek, Fellow, IEEE, and Konstantinos P. Papathanassiou, Fellow, IEEE
}

\begin{abstract}
A novel oriented volume over ground (OVoG) inversion scheme is developed and tested on a data set of simulated agricultural scenarios and real SAR acquisitions. The algorithm makes use of multibaseline measurements to estimate the whole set of the OVoG structural parameters (e.g., crop height, differential extinction between eigenpolarizations, and ground-to-volume ratios) and is significantly robust against nonvolumetric decorrelation contributions. The theoretical assessment points out that, in the dual-baseline case, the vegetation height $h_{V}$ can be estimated with a relative root-mean-square deviation (\% RMSD) of $7.8 \%$ if the selected baselines fulfill the condition $1.2<\kappa_{z} h_{V}<2.8 \mathrm{rad}$ ( $\kappa_{z}$ is the vertical wavenumber). Furthermore, the variance of the estimates is inversely related to the number of baselines $\mathrm{Nb}$. Compared with the dual-baseline case, the RMSD of the differential extinction is reduced by $45 \%$ (from 1.1 to $0.6 \mathrm{~dB} / \mathrm{m}$ ) when $N b=5$ baselines are employed, whereas its mean bias is independent of $N b$. The proposed scheme has been assessed using a set of repeatpass F-SAR acquisitions at L-, C-, and X-band of an agricultural area in Germany. Using two baselines, the height of maize and rape fields is estimated with an average 10\% \% RMSD if the inversion is carried out over L-band acquisitions. On the other hand, when $\mathrm{X}$-band data are employed, one can obtain reliable estimates of wheat and barley height, with a \% RMSD better than $24 \%$. The study also indicates the existence of differential wave propagation effects through maize $\left(\Delta \sigma=\sigma_{\mathrm{VV}}-\sigma_{\mathrm{HH}}\right.$ between 0.7 and $1 \mathrm{~dB} / \mathrm{m})$ and rape $(\Delta \sigma=-0.8 \mathrm{~dB} / \mathrm{m})$ canopies at $L$-band.
\end{abstract}

Index Terms-Agriculture, multibaseline, multifrequency, oriented volume over ground (OVoG), parameter retrieval, polarimetric synthetic aperture radar interferometry (Pol-InSAR).

\section{INTRODUCTION}

$\mathbf{T}$ HE remote monitoring of the status of agricultural vegetation is an important and challenging step toward an improved management of the world resources. While remote sensing techniques are successfully employed for crop clas-

Manuscript received June 10, 2015; revised August 4, 2015, November 2, 2015, and January 18, 2016; accepted March 28, 2016. Date of publication May 6, 2016; date of current version June 1, 2016.

M. Pichierri is with the Institute of Environmental Engineering, Swiss Federal Institute of Technology (ETH), 8093 Zurich, Switzerland (e-mail: pichierri@ifu.baug.ethz.ch).

I. Hajnsek is with the Institute of Environmental Engineering, Swiss Federal Institute of Technology (ETH), 8093 Zurich, Switzerland, and also with the Microwaves and Radar Institute, German Aerospace Center (DLR), 82234 Wessling, Germany (e-mail: hajnsek@ifu.baug.ethz.ch; irena.hajnsek@dlr.de).

K. P. Papathanassiou is with the Microwaves and Radar Institute, German Aerospace Center (DLR), 82234 Wessling, Germany (e-mail: kostas. papathanassiou@dlr.de).

Color versions of one or more of the figures in this paper are available online at http://ieeexplore.ieee.org.

Digital Object Identifier 10.1109/TGRS.2016.2553739 sification and change detection, the quantitative inversion of biophysical and dielectric parameters (e.g., crop height and canopy structure) has, to date, not been fully explored.

Synthetic aperture radar (SAR) has the potential for a unique contribution in monitoring Earth's vegetation, as it senses the vertical distribution of scatterers in the canopy with high spatial resolution and low sensitivity to weather. Polarimetric SAR interferometry (Pol-InSAR) exploits the dependence of the interferometric coherence on polarization to enhance the process of extraction of biophysical parameters from SAR measurements.

This technique, which was first introduced in [1] and [2], has been widely used for estimating forest height and biomass, through the inversion of the random volume over ground (RVoG) model [3]-[7]. The forest is modeled by a two-layer scenario encompassing a cloud of particles with no preferred orientation on top of an impenetrable ground layer. As a result, the ground contribution changes with polarization, whereas the propagation through the vegetation remains polarization independent.

On the other hand, many studies on agricultural vegetation [8]-[13] suggest that the anisotropic effects introduced by the shape and orientation of the stalks may result in a polarizationdependent propagation through the canopy (i.e., nonzero differential extinction and refractivity between volume eigenpolarizations). These studies also highlighted the dependence of such a behavior on the sensor frequency and the radar look angle. As an example, field [13] and laboratory [9] experiments demonstrated the existence of pronounced differential propagation effects for corn plants at L- and S-bands.

For this reason, an oriented volume over ground (OVoG) [14], [15] has been developed to model the scattering from an agricultural scenario. The main constraint in using such a model is the large number of structural parameters with respect to the available measurements. In the single-baseline case, the inverse problem is underdetermined, and appropriate assumptions (e.g., over the ground-to-volume ratios or the wave extinctions) need to be imposed. The validity of such assumptions is subject to the crop signature at certain frequencies and deserves dedicated investigations. For instance, assuming a negligible ground-tovolume ratio at lower frequencies may be still reasonable for rape, but not for rice. Previous studies over agriculture [10], [11] indicated that single-baseline inversion schemes relying on such assumptions might provide, in some cases, incorrect results.

The use of multibaseline measurements allows one to overcome this limitation, as it provides a larger observation space 
[12]. However, as pointed out in [14] and [16], the multibaseline OVoG inverse problem may be ill conditioned, and a small perturbation of the observables can cause a pronounced change in the estimated solution. The structure of the observed scenario certainly affects the degree of such ill conditioning, as well as the choice of the multibaseline configuration. Nevertheless, a comprehensive investigation of such an impact has not yet been addressed in the literature.

The objective of this work is to develop a multibaseline inversion scheme for the estimation of the OVoG structural parameters (e.g., crop height, differential extinction between eigenpolarizations, and ground-to-volume ratios). The proposed methodology makes use of lookup tables computed over a discrete set of height and extinction values to minimize the distance between observables and model predictions.

The algorithm is initially assessed on simulated agricultural scenarios by means of a set of Monte Carlo analyses. A validation on experimental multifrequency SAR acquisitions is then performed, with comparison to in situ measurements. The aim of such a study is to assess the algorithm robustness against nonvolumetric decorrelation sources (e.g., SNR and temporal decorrelation [17]) and its stability over different acquisition geometries and crop structures.

A robust estimation of the $\mathrm{OVoG}$ parameters, such as the extinction coefficients and the ground-to-volume ratios, may help characterize the structure and the dielectric properties of the vegetation, as well as its interaction with polarized waves at different frequencies.

This paper is organized as follows. In Section II, the forward model to be used for the inversion and the sensitivity study is presented. To make use of a general backscattering formulation, the X-Bragg model [18] and the Neumann parametrization [19], [20] are adopted to shape the surface and the direct volume component, respectively. As a result, one can either model an OVoG or an RVoG scenario, so that the sensitivity study is not tuned on a specific vegetation structure. In Section III, the multibaseline inverse problem is discussed, and the modelbased inversion scheme is introduced. In Section IV, the inversion performance is assessed over a collection of simulated SAR data of agricultural scenarios. The sensitivity study intends to ascertain the algorithm robustness against the number of baselines, the choice of the multibaseline configuration, and any changes of the model structural parameters. In Section V, the proposed scheme is then evaluated using a data set of observed multibaseline SAR acquisitions, which were acquired by the German Aerospace Center (DLR)'s F-SAR system at L-, $\mathrm{C}$-, and X-bands over an agricultural area in Germany.

\section{FORWARD MODEL}

The polarimetric/interferometric signatures of agricultural crops are modeled by the OVoG model, to account for the polarization-dependent propagation.

Accordingly, the interaction of the incident wave with the crop canopy and the underlying ground is described by a twolayer scenario whose scattering properties depend upon the nonuniform particles orientation distribution in the vegetation layer. As first derived in [14], the interferometric coherency matrix $\left[\Omega_{O V}\right]$ in the $H-V$ basis for a vegetated layer at a radar incidence angle $\theta$ can be parametrized by

$$
\begin{aligned}
& {\left[\Omega_{O V}\right]=e^{\left[i \phi_{0}-\frac{\left(\sigma_{a}+\sigma_{b}\right) h_{V}}{\cos \theta}\right]}\left[R\left(2 \psi_{V}\right)\right]} \\
& \left\{\int_{0}^{h_{V}} e^{i \kappa_{z} z+\frac{\left(\sigma_{a}+\sigma_{b}\right) z}{\cos \theta}} \cdot[P(z)]\left[T_{O V}^{\prime}\right]\left[P^{\dagger}(z)\right] d z\right\}\left[R\left(-2 \psi_{V}\right)\right] .
\end{aligned}
$$

Equation (1) describes the scattering from a collection of identical particles with a mean orientation $\psi_{V}$, uniformly distributed in a volume layer of depth $h_{V}$. The wave propagation through this anisotropic layer is characterized by a differential (two-way) "effective" extinction $\Delta \sigma=\sigma_{b}-\sigma_{a}$ between volume eigenpolarizations $\vec{p}_{a}$ and $\vec{p}_{b}$. The extinction coefficients $\sigma_{a}$ and $\sigma_{b}$ account for both the wave attenuation through an effective volume medium and the single-scattering loss (i.e., ignoring multiple scattering effects) from the volume particles. The volume eigenpolarizations are defined as two polarization states perpendicular and parallel to the mean particle orientation $\psi_{V}$.

The $[R]$ matrix accounts for the rotation to the $H-V$ polarization basis, i.e.,

$$
[R]=\left[\begin{array}{ccc}
1 & 0 & 0 \\
0 & \cos 2 \psi_{V} & \sin 2 \psi_{V} \\
0 & -\sin 2 \psi_{V} & \cos 2 \psi_{V}
\end{array}\right] .
$$

Furthermore, $[P]$ characterizes the propagation difference (including both the differential extinction $\Delta \sigma$ and refractivity $\Delta \chi=\chi_{b}-\chi_{a}$ effects) of the two volume eigenpolarizations [14] and is defined as follows:

$$
[P]=\left[\begin{array}{ccc}
\cosh \zeta & \sinh \zeta & 0 \\
\sinh \zeta & \cosh \zeta & 0 \\
0 & 0 & 1
\end{array}\right]
$$

where

$$
\zeta=\left(\frac{\Delta \sigma}{2}+i k_{0} \Delta \chi\right) \frac{z-h_{V}}{\cos (\theta)}
$$

Finally, the ground phase term $\phi_{0}=\kappa_{z} z_{0}$ refers to the product of the vertical wavenumber $\kappa_{z}$ and the height of the ground (with respect to a reference) $z_{0}$.

The volume polarimetric coherency matrix $\left[T_{O V}^{\prime}\right]$ in (1) is reflection symmetric about the eigenpolarizations. A possible parametrization of the aforementioned matrix was proposed in [19], where the scattering from the vegetation canopy is modeled under the distorted Born approximation by a homogeneous layer of spheroidal particles whose orientation follows a circular Gaussian distribution. As a result, $\left[T_{O V}^{\prime}\right]$ is expressed as a function of three parameters: the real and imaginary parts of the particle anisotropy $\delta(|\delta| \in[0,1])$ and the normalized degree of randomness $\tau$. The former account for the effective shape of the scatterer, whereas the latter is related to the standard deviation of the particles orientation distribution $(\tau=0$ when the scatterers are strongly $\psi_{V}$ oriented, whereas it tends to 1 for randomly distributed particles). For further details, the readers are referred to [7] and [19]. 
Similar to the volume component, one can derive an expression for the interferometric coherency matrix $\left[\Omega_{S}\right]$ of the underlying ground layer [21]. By assuming a surface main orientation $\psi_{S}$ and an altitude of $z_{0}$

$$
\begin{array}{r}
{\left[\Omega_{S}\right]=e^{\left[i \phi_{0}-\frac{\left(\sigma_{a}+\sigma_{b}\right) h_{V}}{\cos \theta}\right]}\left[R\left(2 \psi_{V}\right)\right][P(0)][R(-2 \Delta \psi)]\left[T_{S}^{\prime}\right]} \\
\cdot[R(2 \Delta \psi)]\left[P^{\dagger}(0)\right]\left[R\left(-2 \psi_{V}\right)\right]
\end{array}
$$

with $\Delta \psi=\psi_{V}-\psi_{S}$.

The ground polarimetric coherency matrix $\left[T_{S}^{\prime}\right]$ used in the following is derived under the X-Bragg model [18], so that the sensitivity study can be applied over a wide range of surface roughness conditions.

Under the hypothesis of statistically uncorrelated scattering mechanisms, the OVoG interferometric coherency matrix $\left[\Omega_{\mathrm{OVoG}}\right]$ is defined by the linear combination of the aforementioned ground and volume contributions, i.e.,

$$
\left[\Omega_{\mathrm{OVoG}}\right]=m_{S}\left[\Omega_{S}\right]+m_{V}\left[\Omega_{\mathrm{OV}}\right]
$$

whereas the polarimetric coherency matrix $\left[T_{\mathrm{OVoG}}\right]$ is given by

$$
\left[T_{\mathrm{OVoG}}\right]=m_{S}\left[T_{S}\right]+m_{V}\left[T_{\mathrm{OV}}\right] .
$$

In (7), $T_{S}$ and $T_{\mathrm{OV}}$ are computed from (5) and (1), respectively, in the limit of zero baseline length. Furthermore, $m_{S}$ and $m_{V}$ represent the strength of the ground and volume contributions, respectively.

By assuming polarimetric stationarity and equal polarizations at baseline ends, the resulting Pol-InSAR OVoG complex coherence $\gamma_{\text {OVoG }}$ [1] can be expressed as

$$
\gamma_{\mathrm{OVoG}}(\vec{\omega})=\frac{\vec{\omega}^{\dagger}\left[\Omega_{\mathrm{OVoG}}\right] \vec{\omega}}{\vec{\omega}^{\dagger}\left[T_{\mathrm{OVOG}}\right] \vec{\omega}}=e^{i \phi_{0}} \frac{\gamma_{O V}(\vec{\omega})+\mu(\vec{\omega})}{1+\mu(\vec{\omega})} .
$$

In (8), the normalized projection vectors $\vec{\omega}$ correspond to the copolarizations and the cross-polarization in the eigenpolarization basis. The ground-to-volume scattering ratio $\mu \in \mathbb{R}$ and the volume decorrelation $\gamma_{\mathrm{OV}}$ are given by

$$
\begin{aligned}
\mu(\vec{\omega}) & =\frac{2 \sigma(\vec{\omega})}{\cos \theta\left(e^{2 \sigma(\vec{\omega}) h_{V} / \cos \theta}-1\right)} \frac{\vec{\omega}^{\dagger}\left[T_{S}^{\prime}\right] \vec{\omega}}{\vec{\omega}^{\dagger}\left[T_{\mathrm{OV}}^{\prime}\right] \vec{\omega}} \geq 0 \\
\gamma_{\mathrm{OV}}(\vec{\omega}) & =\frac{\int_{0}^{h_{V}} e^{\frac{2 \sigma(\vec{\omega})}{\cos \theta} z} e^{i \kappa_{z} z} d z}{\int_{0}^{h_{V}} e^{\frac{2 \sigma(\vec{\omega})}{\cos \theta} z} d z} .
\end{aligned}
$$

In contrast to the RVoG case, the extinction $\sigma$ and, thus, $\gamma_{\mathrm{OV}}$, are now polarization dependent. More importantly, the extinction in the cross-polarized channel is defined by the mean of the corresponding copolarized extinction values.

If the system acquires in a repeat-pass configuration or in a single-pass alternate-transmit mode, then (8) is formally valid also in the presence of a significant dihedral response (e.g., ground-stalk interaction). In this case, the parameter $\mu$ in (8) will now account for both the direct surface and the dihedral scattering contributions (a formulation of $\mu$ was derived in [23]). On the other hand, in the single-pass single-transmit mode, this additional dihedral ground-stalk response must be modeled via an additional decorrelation term [15], [22], [23].
The knowledge of the volume eigenpolarizations is not straightforward, as the mean orientation angles of the volume and ground are unknown. For the sake of simplicity, in the following, the ground mean orientation angle is assumed to be zero, whereas the particles within the vegetation canopy are assumed to be vertically oriented.

\section{OVOG INVERSION SCHEME}

If the volume eigenpolarization basis is known, a fully polarimetric single-baseline SAR acquisition allows computing the complex coherences for the two eigenpolarizations and the corresponding cross-polarized channel, providing a set of three independent complex observables that can be parametrized by (8).

On the other hand, the OVoG model is fully described by seven parameters: the topographic phase $\phi_{0}$, the vegetation height $h_{V}$, the extinctions for the two eigenpolarizations, and the ground-to-volume ratios for the three polarimetric channels. Therefore, the OVoG inverse problem is in general underdetermined if only one baseline is employed. In this case, a priori information (e.g., the precise knowledge of the underlying topography) or further simplifying assumptions (e.g., pure volume coherence, or a negligible differential extinction) have to be used to obtain a balanced inverse problem.

A multibaseline approach can potentially circumvent this limitation, if the OVoG parameters are assumed to be invariant with respect to the vertical wavenumber $\kappa_{z}$ and the impact of temporal decorrelation [17], [24] is ignored. If a set of $N b \geq 2$ phase calibrated baselines is available, then the number of observables increases by a factor of $N b$, whereas that of the unknown parameters remains the same. Consequently, one can take advantage of the expanded observation space to retrieve the whole set of the OVoG parameters, via a mathematical optimization. In a previous study [12], this was achieved (for the dual-baseline case) by minimizing the distance between the modeled and measured coherences, via a genetic algorithm optimization.

The proposed inversion technique makes use of $N b \geq 2$ baselines and provides a unique estimate of the OVoG parameters by means of an exhaustive search over a predefined solution space of extinctions and vegetation heights. Together with the invariance of the model parameters with respect to $\kappa_{z}$, one must assume that the ground scattering center for a fixed baseline is independent of polarization, which means that the two eigenpolarizations and the cross-polarized channel share the same ground phase.

The algorithm also requires the knowledge of the volume eigenpolarizations. If the chosen eigenpolarization basis differs from the actual one, it may happen that the relation between the extinctions described in Section II is not valid as (8) cannot be used to model the cross- and copolarized complex coherences.

For the sake of interpretation, the inversion scheme is split into four steps and described in the following. Please note that the $A-B$ linear basis is chosen as the general volume eigenpolarization basis. Thus, the notations $A A$ and $B B$ identify the copolarized channels, whereas $A B$ is the cross-polarized one. The subscripts $p$ and $q$ refer to the receive and transmit 
polarizations $(p, q)=\{(A, A),(B, B),(A, B)\}$. Furthermore, lowercase subscripts are used for the intermediate estimates, whereas the subscripts of the final estimates are in capital letters.

\section{A. Step 1}

According to (8), the OVoG complex coherence can be equivalently written as

$$
\gamma_{\mathrm{OVOG}}=e^{i \phi_{0}}\left[\gamma_{\mathrm{OV}}+L\left(1-\gamma_{\mathrm{OV}}\right)\right]
$$

where

$$
L(\vec{\omega})=\frac{\mu(\vec{\omega})}{1+\mu(\vec{\omega})} \geq 0 .
$$

The magnitude squared of $\gamma_{\mathrm{OVoG}}$ is then computed as follows:

$$
\begin{aligned}
\left|\gamma_{\text {OVoG }}\right|^{2}=(1 & \left.+\left|\gamma_{\text {OV }}\right|^{2}-2 \operatorname{Re}\left\{\gamma_{\text {OV }}\right\}\right) L^{2} \\
& +\left(2 \operatorname{Re}\left\{\gamma_{\text {OV }}\right\}-\left|\gamma_{\text {OV }}\right|^{2}\right) L+\left|\gamma_{\text {OV }}\right|^{2} .
\end{aligned}
$$

For each element of the $\left(h_{v}, \sigma_{p q}\right)$ space, a quadratic equation in the variable $L_{p q}=L\left(\vec{\omega}_{p q}\right)$ can be derived from (13) for the $i$ th baseline, i.e.,

$$
f_{i}\left(h_{v}, \sigma_{p q} ; L_{p q}\right)=a_{i} L_{p q}^{2}+b_{i} L_{p q}+c_{i}=0
$$

where

$$
\begin{aligned}
& a_{i}\left(h_{v}, \sigma_{p q}, \kappa_{z, i}\right)=1+\left|\gamma_{\mathrm{OV}, \mathrm{i}}\right|^{2}-2 \operatorname{Re}\left(\gamma_{\mathrm{OV}, \mathrm{i}}\right) \\
& b_{i}\left(h_{v}, \sigma_{p q}, \kappa_{z, i}\right)=2\left[\operatorname{Re}\left(\gamma_{\mathrm{OV}, \mathrm{i}}\right)-\left|\gamma_{\mathrm{OV}, \mathrm{i}}\right|^{2}\right] \\
& c_{i}\left(h_{v}, \sigma_{p q}, \kappa_{z, i}\right)=\left|\gamma_{\mathrm{OV}, \mathrm{i}}\right|^{2}-\left|\gamma_{\mathrm{OVoG}, i}\right|^{2} .
\end{aligned}
$$

In (15), $\gamma_{\mathrm{OVoG}, i}=\gamma_{\mathrm{OVoG}}\left(\vec{\omega}_{p q}, \kappa_{z, i}\right)$ is the measured coherence, whereas the term $\gamma_{O V, i}$ is computed from (10) using the $\left(h_{v}, \sigma_{p q}\right)$ solution space and $\kappa_{z, i}$.

For each of the three polarization channels, a ground-tovolume ratio pair $\left(\mu_{p q}^{(1)}, \mu_{p q}^{(2)}\right)$ is uniquely related to a $\left(h_{v}, \sigma_{p q}\right)$ element of the solution space. This is achieved by solving the following quadratic equation in the variable $L_{p q}$ :

$$
\begin{aligned}
f\left(h_{v}, \sigma_{p q} ; L_{p q}\right) & =\sum_{i}^{N b} f_{i}\left(h_{v}, \sigma_{p q} ; L_{p q}\right) \\
& =\left(a_{1}+\cdots+a_{N b}\right) L_{p q}^{2} \\
& +\left(b_{1}+\cdots+b_{N b}\right) L_{p q}+\left(c_{1}+\cdots+c_{N b}\right)=0 .
\end{aligned}
$$

Failure Case: As $L_{p q}$ must be a nonnegative real number, all $\left(h_{v}, \sigma_{p q}\right)$ elements for which both solutions to (16) are imaginary or real negative are discarded.

\section{B. Step 2}

The ground phase solution pair of the $i$ th baseline $\left(\phi_{p q, i}^{(1)}\right.$, $\left.\phi_{p q, i}^{(2)}\right)$ is then computed from (8), i.e.,

$$
\phi_{p q, i}^{(m)}=\arg \left(\frac{\gamma_{\mathrm{OVoG}, i}\left(1+\mu_{p q}^{(m)}\right)}{\gamma_{O V, i}+\mu_{p q}^{(m)}}\right)
$$

where $m=1,2$.

\section{Step 3}

A set of $\sigma$ triplets $\left[\Sigma_{a a}\left(h_{v}\right), \Sigma_{b b}\left(h_{v}\right), \Sigma_{a b}\left(h_{v}\right)\right]$ is computed as a function of $h_{v}$. The following strategy is replicated for each element of the $h_{v}$ solution space.

- Starting from (17), two lookup tables are computed for a given polarization. Assuming that the crosspolarized channel $A B$ is chosen, then two vectors $\Phi^{(1)}=$ $\left[\begin{array}{lll}\phi_{a b, 1}^{(1)} & \cdots & \phi_{a b, N b}^{(1)}\end{array}\right]$ and $\Phi^{(2)}=\left[\begin{array}{lll}\phi_{a b, 1}^{(2)} & \cdots & \phi_{a b, N b}^{(2)}\end{array}\right]$ are associated with each element of the $\sigma_{a b}$ solution space.

- Equation (17) is inverted for each of the two remaining polarizations (i.e., the extinction coefficients $\sigma_{a a, i}$ and $\sigma_{b b, i}$ are now expressed as a function of the ground phase $\phi \in[-\pi, \pi])$. Note that the magnitude of the volume decorrelation $\gamma_{\mathrm{OV}, i}$, at a given $\phi$ and $h_{v}$, increases monotonically with the extinction $\sigma_{p q}$. Therefore, one may associate a unique value of $\sigma_{a a, i}$ and $\sigma_{b b, i}$ (if they exist) with each $\phi$ in the interval $[-\pi, \pi]$ (i.e., in the unit circle, there is a single intersection between the curve $\gamma_{\mathrm{OV}, i}\left(\sigma_{p q}\right)$ and the line connecting the ground phase $\phi$ to $\left.\gamma_{\mathrm{OVoG}, i}\left(\vec{\omega}_{p q}\right)\right)$. As a result, $N b \sigma$-pairs $\left(\sigma_{a a, i}(\phi), \sigma_{b b, i}(\phi)\right)$ are generated.

- As the extinction coefficients do not depend upon $\kappa_{z}$, the best $\sigma$ triplet $\left[\Sigma_{a a}, \Sigma_{b b}, \Sigma_{a b}\right]$ is finally obtained by minimizing the diversity of the $\sigma$ pairs $\left(\sigma_{a a, i}(\phi), \sigma_{b b, i}(\phi)\right)$ over the $N b$ baselines, given that all polarizations share the same ground phase. Such a minimization is achieved by means of the following criterion:

$\left(\Sigma_{a b} ; M\right)= \begin{cases}\left(\Sigma_{a b}^{(1)} ; 1\right), & \text { if } \min _{\sigma_{a b}}\left\{J^{(1)}\right\} \leq \min _{\sigma_{a b}}\left\{J^{(2)}\right\} \\ \left(\Sigma_{a b}^{(2)} ; 2\right), & \text { otherwise }\end{cases}$ where $(m=1,2)$

$$
\begin{aligned}
& \Sigma_{a b}^{(m)}=\underset{\sigma_{a b}}{\arg \min } J^{(m)} \\
&=\underset{\sigma_{a b}}{\arg \min }\left\{\sum_{i}^{N b}\left[\sigma_{a a, i}\left(\phi_{a b, i}^{(m)}\right)-\bar{\sigma}_{a a}\right]^{2}\right. \\
&\left.\quad+\sum_{i}^{N b}\left[\sigma_{b b, i}\left(\phi_{a b, i}^{(m)}\right)-\bar{\sigma}_{b b}\right]^{2}\right\} \\
& \bar{\sigma}_{a a}=\frac{1}{N b} \sum_{i}^{N b} \sigma_{a a, i}\left(\phi_{a b, i}^{(m)}\right) \\
& \bar{\sigma}_{b b}=\frac{1}{N b} \sum_{i}^{N b} \sigma_{b b, i}\left(\phi_{a b, i}^{(m)}\right) .
\end{aligned}
$$

At the end of Step 3, the $\sigma$ triplets $\left[\Sigma_{a a}, \Sigma_{b b}, \Sigma_{a b}\right]$ are retrieved as a function of $h_{v}$, where $\Sigma_{a a}=\bar{\sigma}_{a a}\left(\phi_{a b, i}^{(M)}\left(\Sigma_{a b}\right)\right)$, and $\Sigma_{b b}=\bar{\sigma}_{b b}\left(\phi_{a b, i}^{(M)}\left(\Sigma_{a b}\right)\right)$.

Failure Case: For a given $h_{v}$, it may happen that there are no values of $\phi_{a b, i}^{(m)}(\forall m=1,2)$ for which both $\sigma_{a a, i}$ and $\sigma_{b b, i}$ are defined. In this case, the preceding minimization cannot be computed, and that particular $h_{v}$ solution is discarded. 
TABLE I

Maize Scenario: Simulated Parameters

\begin{tabular}{cc}
\hline \multicolumn{2}{c}{ Oriented Volume } \\
\hline \hline Particle Anisotropy $|\delta|$ & 0.4 \\
Particle Orientation $\psi_{V}[\mathrm{deg}]$ & Vertical \\
VV Extinction $\sigma_{V V}[\mathrm{~dB} / \mathrm{m}]$ & 1 \\
HH Extinction $\sigma_{H H}[\mathrm{~dB} / \mathrm{m}]$ & 0.25 \\
Vegetation Height $h_{V}[\mathrm{~m}]$ & 1.7 \\
Degree of Randomness $\tau$ & 0.65 \\
\hline \hline Surface \\
\hline \hline Surface permittivity & $20-2 i$ \\
Ground altitude $z_{0}[\mathrm{~m}]$ & 0 \\
X-Bragg's $\beta_{1}[\mathrm{rad}]$ & $\pi / 2$ \\
\hline
\end{tabular}

TABLE II

ANY-CRop ScEnario: Simulated Parameters

\begin{tabular}{cc}
\hline \multicolumn{2}{c}{ Oriented Volume } \\
\hline \hline Particle Anisotropy $|\delta|$ & {$[0.2,0.9]$} \\
Particle Orientation $\psi_{V}[\mathrm{deg}]$ & Vertical \\
Differential Extinction $\Delta \sigma[\mathrm{dB} / \mathrm{m}]$ & {$[0.1,1.5]$} \\
HH Extinction $\sigma_{H} H[\mathrm{~dB} / \mathrm{m}]$ & {$[0.05,1]$} \\
Vegetation Height $h_{V}[\mathrm{~m}]$ & {$[1,2.5]$} \\
Degree of Randomness $\tau$ & {$[0.3,0.8]$} \\
Normalized Volume Power NVP & {$[0.2,0.95]$} \\
\hline \hline \multicolumn{2}{c}{ Surface } \\
\hline \hline Surface permittivity (Real) & {$[15,25]$} \\
Surface permittivity (Imaginary) & {$[-5,-1]$} \\
Ground altitude $z_{0}[\mathrm{~m}]$ & 0 \\
X-Bragg's $\beta_{1}[\mathrm{rad}]$ & {$[\pi / 8, \pi / 2]$} \\
\hline
\end{tabular}

\section{Step 4}

The final solution $\left(h_{V} ; \sigma_{A A}, \sigma_{B B}, \sigma_{A B}\right)$ can be estimated from the set of $\sigma$ triplets in Step 3.

As aforementioned in Section II, the OVoG model defines the extinction $\sigma_{A B}$ in the cross-polarized channel by the average of the copolar extinctions $\sigma_{A A}$ and $\sigma_{B B}$.

By defining $\Sigma_{a b \text {,synt }}\left(h_{v}\right)=\left(\Sigma_{a a}\left(h_{v}\right)+\Sigma_{b b}\left(h_{v}\right)\right) / 2$, the estimated height $h_{V}$ is given by

$$
h_{V}=\underset{h_{v}}{\arg \min }\left|\Sigma_{a b, \mathrm{synt}}\left(h_{v}\right)-\Sigma_{a b}\left(h_{v}\right)\right|
$$

whereas the estimated extinctions are $\sigma_{A A}=\Sigma_{a a}\left(h_{V}\right), \sigma_{B B}=$ $\Sigma_{b b}\left(h_{V}\right)$, and $\sigma_{A B}=\Sigma_{a b}\left(h_{V}\right)$.

\section{Simulated Data Analysis}

Here, a set of Monte Carlo simulations are conducted to assess the performance of the proposed algorithm against $\kappa_{z}$, as well as its sensitivity to variations of the OVoG structural parameters.

The performance analysis is carried out for two different scenarios:

- maize, where the OVoG parameters are kept fixed to reproduce a typical maize scenario;

- any-crop, where the OVoG parameters are randomly selected within predefined intervals.

The parameters used to generate the maize and any-crop scenarios are shown in Tables I and II, respectively. The acquisition parameters are summarized in Table III.
TABLE III

ACQuisition System PaRAmeters of THE Monte CaRlo Analysis

\begin{tabular}{cc}
\hline \multicolumn{2}{c}{ System variables } \\
\hline \hline Wavelength [m] & 0.23 \\
Chirp BW [MHz] & 150 \\
Sensor altitude [km] & 2.4 \\
Incidence angle [deg] & 40 \\
\hline
\end{tabular}

For each element of the two scenarios, a corresponding Pol-InSAR realization is simulated by using the following approach.

- The interferometric coherency matrix $\left[\Omega_{\mathrm{OVOG}}\right]$ and the polarimetric coherency matrix $\left[T_{\mathrm{OVOG}}\right]$ are computed from the selected $\mathrm{OVoG}$ parameters as described in Section II.

- Under the hypothesis of fully developed speckle, the radar observations are then modeled as random variables $\left[\Omega_{\mathrm{OVoG}}^{(L)}\right]$ and $\left[T_{\mathrm{OVoG}}^{(L)}\right]$ following the complex Wishart distribution (where $L$ is the number of independent looks). Unless otherwise specified, $L=225$.

- Finally, the simulated realization consists of $N=250$ independent samples from this (Wishart) distribution.

To measure the performance of the inversion scheme, the root-mean-square deviation RMSD and the mean bias deviation MBD and their relative values (\%RMSD and \%MBD) are used, i.e.,

$$
\begin{aligned}
\mathrm{RMSD} & =\sqrt{\frac{\sum_{i=1}^{N_{c}}\left(x_{i}-x_{\text {true }}\right)^{2}}{N_{c}}} \\
\% \mathrm{RMSD} & =100 \cdot \frac{\mathrm{RMSD}}{x_{\text {true }}} \\
\mathrm{MBD} & =\frac{\sum_{i=1}^{N_{c}}\left(x_{i}-x_{\text {true }}\right)}{N_{c}} \\
\% \mathrm{MBD} & =100 \cdot \frac{\mathrm{MBD}}{x_{\text {true }}} .
\end{aligned}
$$

In (22) and (24), $N_{c}$ is the number of realization's independent samples for which a valid solution exists, whereas $x_{i}$ and $x_{\text {true }}$ are the estimated and actual values, respectively.

The RMSD and the MBD provide a quantitative measure of the estimation error for each simulated realization. Therefore, as a rule of thumb, the smaller the values (in magnitude) are, the closer is the retrieved outcome to the true one. Note that, if the algorithm does not provide a valid solution for at least $75 \%$ of the realization's independent samples, then the MBD and the RMSD are not computed and the realization is discarded.

The $\left(h_{V}, \sigma\right)$ pair is estimated within a predefined range of extinctions (consistent with measured values from [13]) and vegetation heights. In particular, the maximum extinction is set to $4.5 \mathrm{~dB} / \mathrm{m}$, whereas $h_{V, \max }=3.5 \mathrm{~m}$.

With regard to the search space for the ground phase, two scenarios have been investigated. In the first scenario, the inversion scheme is carried out on the full-range of ground phase values (set to $[-\pi, \pi]$ by default). In the second one, the search space for the ground phase of the $i$ th baseline is constrained to the interval $\left[\phi_{p q, i}-\kappa_{z, i} \frac{\Delta z}{2}, \phi_{p q, i}+\kappa_{z, i} \frac{\Delta z}{2}\right]$, centered at the actual ground phase $\phi_{p q, i}=0$, where $\Delta z$ is the extent 


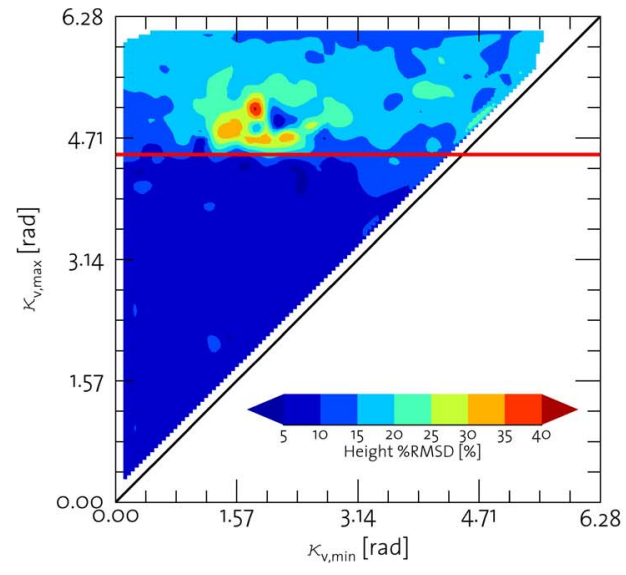

(a)

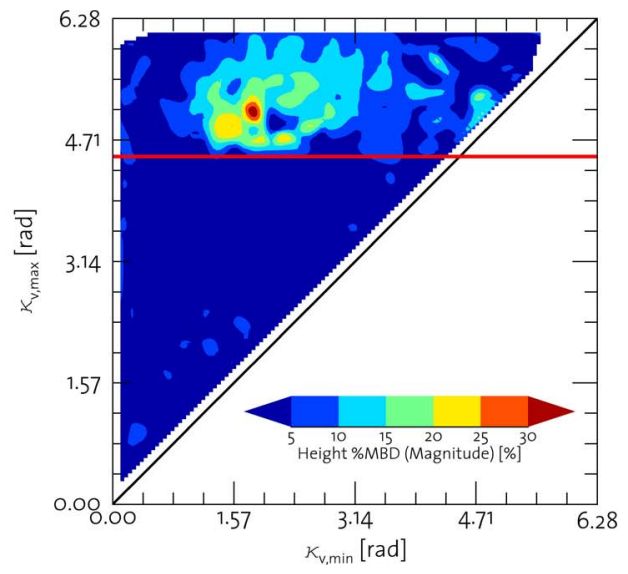

(b)

Fig. 1. Height (a) $\%$ RMSD and (b) $|\% \mathrm{MBD}|$ in the dual-baseline case: the two axes refer to the realizations' minimum and maximum $\kappa_{v}$ (in rad). The red threshold is set at $\kappa_{v, \max }=4.5 \mathrm{rad}$.

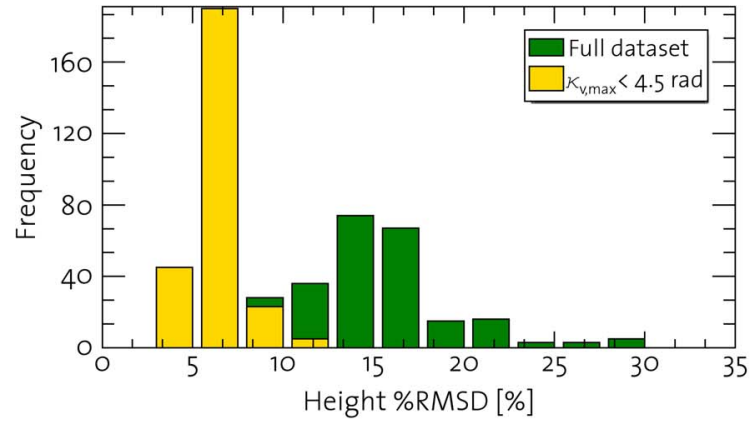

(a)

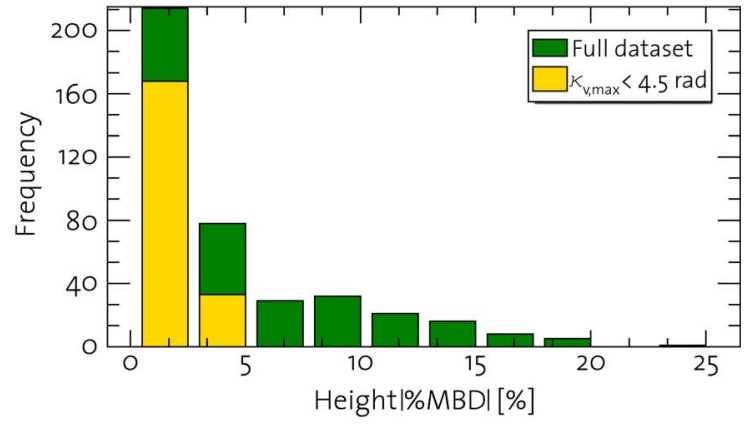

(b)

Fig. 2. Height (a) \%RMSD and (b) $|\% \mathrm{MBD}|$ in the dual-baseline case: the histogram of the full data set is in green; the histogram of the realizations whose $\kappa_{v, \max }<4.5 \mathrm{rad}$ is in yellow.

of the search space for the ground height, and $\kappa_{z, i} \frac{\Delta z}{2}<\pi$. Equivalently, $\Delta z$ represents how accurate is the a priori knowledge of the ground height.

For the sake of interpretation, the first approach will be referred to as full-range in the following. Where the algorithm is carried out over a $\phi_{p q, i}$ interval, the width of the interval will be specified by its $\Delta z$.

\section{A. Maize Scenario: Assessment Over Changes of $\kappa_{z}$}

This first assessment aims at understanding the impact of the vertical wavenumber $\kappa_{z}$ on the inversion performance.

Initially, a Monte Carlo analysis is performed over a total of 500 realizations of the maize scenario. For each of them, $\mathrm{Nb}=$ 2 spatial baselines $B_{i}$ are randomly generated such that the associated $\kappa_{v, i}=\kappa_{z, i} h_{V}$ values vary over the interval $[0,2 \pi]$ and $\Delta \kappa_{v}=\left|\kappa_{v, 1}-\kappa_{v, 2}\right|>0.2 \mathrm{rad}$. The $m_{V} / m_{S}$ ratio is set to 2.4 , and $\Delta z=0.4 \mathrm{~m}$.

The height inversion results are shown in Fig. 1: a 2-D plot is drawn, where the two axes refer to $\kappa_{v \text {,min }}$ and $\kappa_{v \text {,max }}$, namely, the minimum and maximum $\kappa_{v}$ of the realizations, respectively. The color is related to the interpolated values of the \% RMSD and the $\% \mathrm{MBD}$ of the estimated height.

To some extent, remarkable dependence of the inversion performance on the maximum $\kappa_{z}$ is found: as one moves along the $\kappa_{v, \text { max}}$-axis, a region can be easily identified where the height \%RMSD remains below $10 \%$. This area encompasses the realizations whose $\kappa_{v, \max }$ is lower than approximately $4.5 \mathrm{rad}$ [see Fig. 2(a)]. On the other hand, the statistics suggest a performance degradation ( $\% \mathrm{RMSD}=32 \%$, in the worst case) when larger $\kappa_{z}$ are employed. Note that, in the region where $\kappa_{v, \max }<4.5 \mathrm{rad}$, the inversion performance seems rather insensitive to changes of $\kappa_{v \text {, min }}$.

Such dependence upon $\kappa_{z}$ is also found for the estimated differential extinction (see Figs. 3 and 4). Within the aforementioned boundary, the retrieved $\Delta \sigma$ is consistent with the actual one $(|\mathrm{MBD}|<0.25 \mathrm{~dB} / \mathrm{m}$ for $95 \%$ of the realizations $)$, whereas the mismatch between the real and estimated values is likely to increase (maximum $|\mathrm{MBD}|=1.15 \mathrm{~dB} / \mathrm{m}$ ) if one considers the realizations with $\kappa_{v, \max }>4.5 \mathrm{rad}$.

When the distribution of the height \%RMSD is displayed as a function of $\kappa_{v, \max }$ [see Fig. 5(a)], one may observe that the median of the distribution is approximately below $10 \%$ if $\kappa_{v, \text { max }}<4.5 \mathrm{rad}$, whereas its value increases as one moves toward high $\kappa_{v, \max }$ values. A qualitative comparison of the \%RMSD's median with the Pol-InSAR coherences $\left|\gamma_{\mathrm{OVOG}}\right|$ computed for the three polarimetric channels [see Fig. 5(b)] indicates that the performance degradation for large $\kappa_{z}$ may be related to the presence of a strong volume decorrelation $\left(\left|\gamma_{\text {OVoG }}\right|<0.4\right.$ when $\left.\kappa_{v, \text { max }}>4.5 \mathrm{rad}\right)$. 


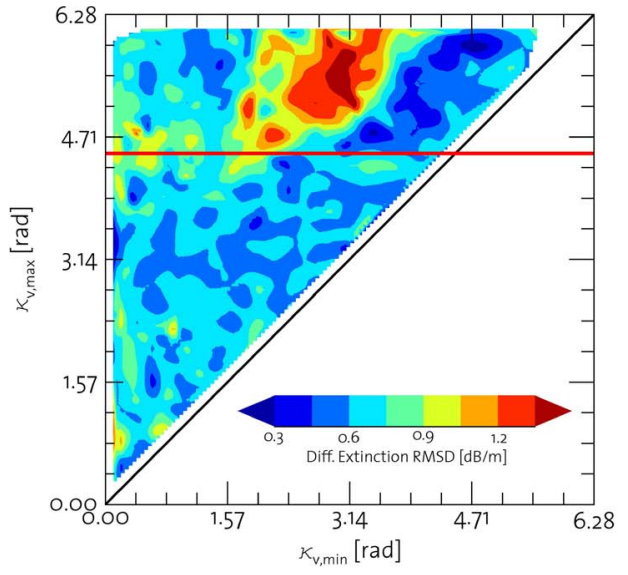

(a)

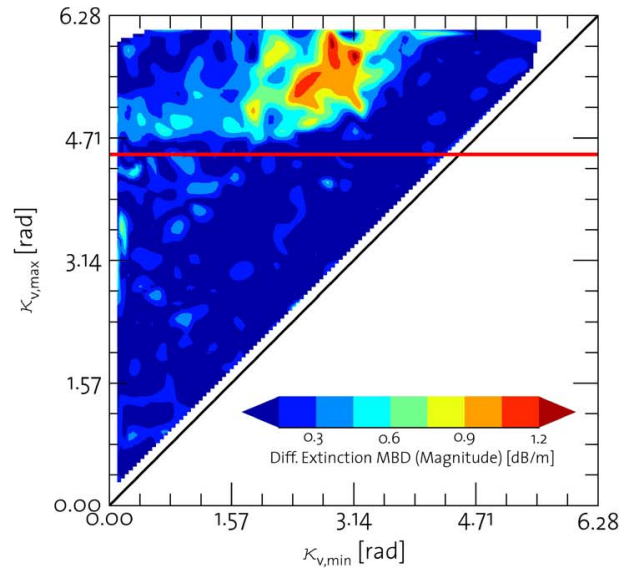

(b)

Fig. 3. Differential extinction (a) RMSD and (b) $|\mathrm{MBD}|$ in the dual-baseline case: the two axes refer to the realizations' minimum and maximum $\kappa_{v}$ (in rad). The red threshold is set at $\kappa_{v, \max }=4.5 \mathrm{rad}$.

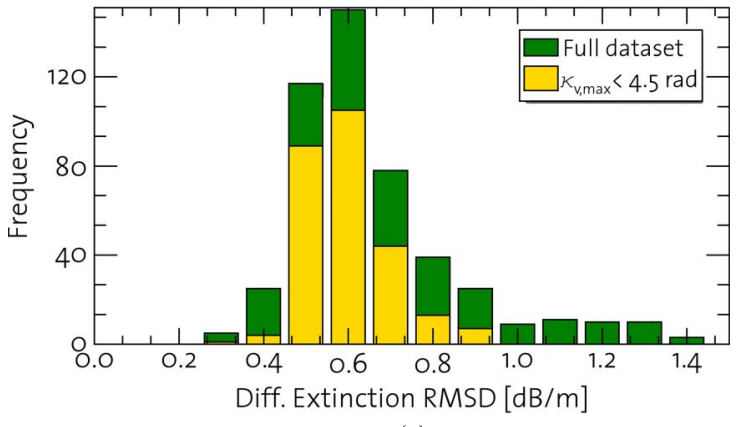

(a)

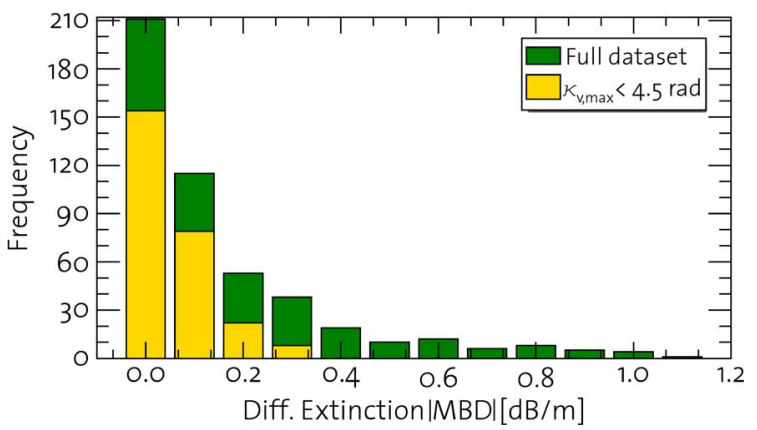

(b)

Fig. 4. Differential extinction (a) RMSD and (b) $|\mathrm{MBD}|$ in the dual-baseline case: the histogram of the full data set is in green; the histogram of the realizations whose $\kappa_{v, \max }<4.5 \mathrm{rad}$ is in yellow.

A Monte Carlo analysis is also carried out in order to ascertain the algorithm robustness against additional losses in the Pol-InSAR coherence (e.g., SNR decorrelation or temporal decorrelation in repeat-pass interferometry). A total of 500 realizations of the maize scenario for $N b=2$ is generated, where the complex coherence (for the $i$ th baseline) is now modeled as the product formed from the OVoG coherence $\left[\gamma_{\mathrm{OVOG}, i}\right.$; see (8)] and a scalar decorrelation factor $\gamma_{\mathrm{dec}, i}$.

Figs. 6-9 display the results for $\gamma_{\mathrm{dec}, 1}=\gamma_{\mathrm{dec}, 2}=0.95$ and $\Delta z=0.4 \mathrm{~m}$. The histograms show an overall degradation of the level of accuracy, as now only $18 \%$ of the realizations with $\kappa_{v, \max }<4.5 \mathrm{rad}$ have a height $\%$ RMSD below $10 \%$. At first

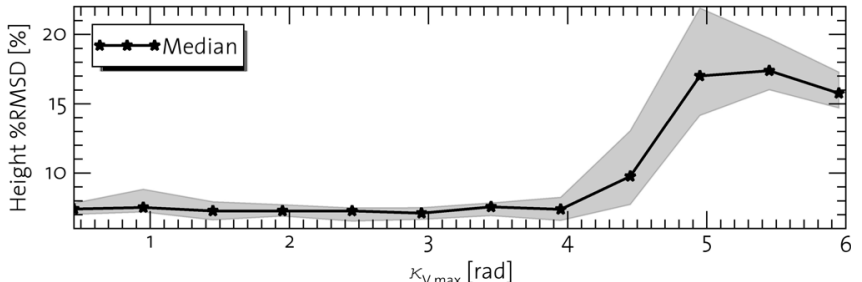

(a)

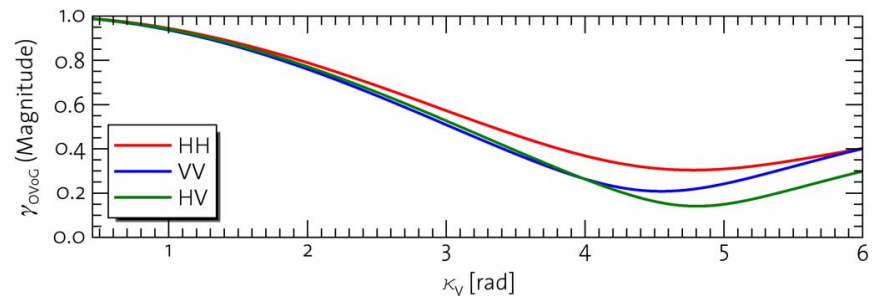

(b)

Fig. 5. (a) Height $\%$ RMSD distribution as a function of $\kappa_{v, \max }$ [the solid line is the median, whereas the black region is the interquartile range (IQR)]. (b) $\left|\gamma_{O V o G}\right|$ as a function of $\kappa_{v}$, for the three polarimetric channels: $\mathrm{HH}$ (red), VV (blue), and HV (green).

glance, one may observe that, now, the algorithm performance undergoes a pronounced degradation for smaller $\kappa_{z}$. Furthermore, a negative correlation of the statistics with $\kappa_{v, \text { max }}$ is noticeable. The 2-D plots in Figs. 6 and 8 also indicate that the algorithm does not return a valid solution when both $\kappa_{v, \text { min }}$ and $\kappa_{v \text {,max }}$ are very small (e.g., below $0.6 \mathrm{rad}$ ), as well as when the difference between the two $\kappa_{v}$ values is approximately larger than $\pi \mathrm{rad}$.

Fig. 10(a) shows the distribution of the height \%RMSD as a function of $\kappa_{v, \text { min }}$ when $\gamma_{\mathrm{dec}, 1}=\gamma_{\mathrm{dec}, 2}=0.95$. Interestingly, an inverse relationship is found between the \%RMSD's median trend and the partial derivative $\left|\frac{\partial\left|\gamma_{\mathrm{OV} G \mathrm{G}}\right|}{\partial \kappa_{v}}\right|$, as shown in Fig. 10(b): in the presence of additional decorrelation sources, a significant estimation error is likely to arise if the sensitivity of the $\mathrm{OVoG}$ coherence to $\kappa_{v}$ is remarkably low (as for small $\kappa_{z}$ ); thus, small variations of the measured coherence strongly affect the accuracy of the retrieved height. 


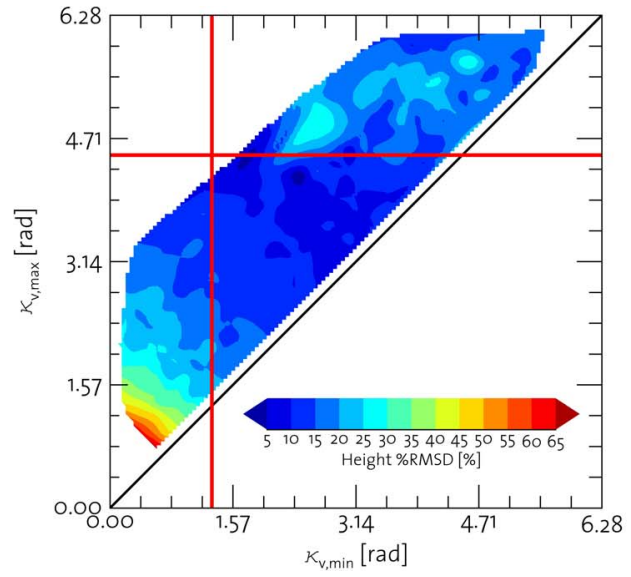

(a)

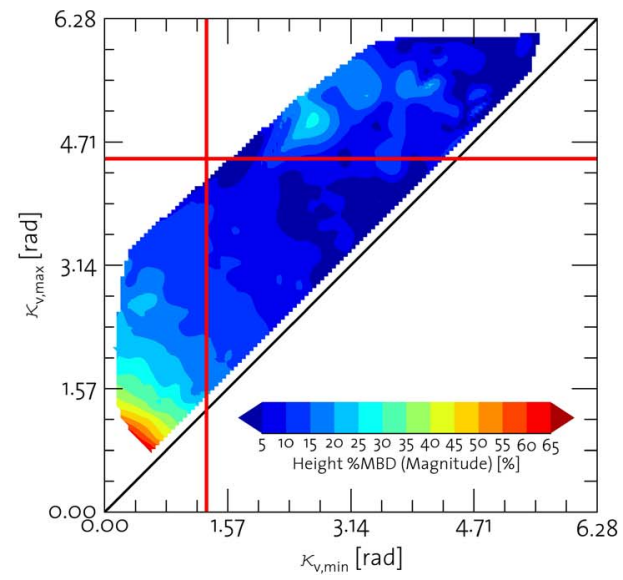

(b)

Fig. 6. Height (a) \%RMSD and (b) $|\% \mathrm{MBD}|$ in the dual-baseline case, with $\gamma_{\mathrm{dec}, 1}=\gamma_{\mathrm{dec}, 2}=0.95$ : the two axes refer to the realizations' minimum and maximum $\kappa_{v}$ (in rad). Two red thresholds are set at $\kappa_{v, \max }=4.5 \mathrm{rad}$ and $\kappa_{v, \min }=1.2 \mathrm{rad}$.

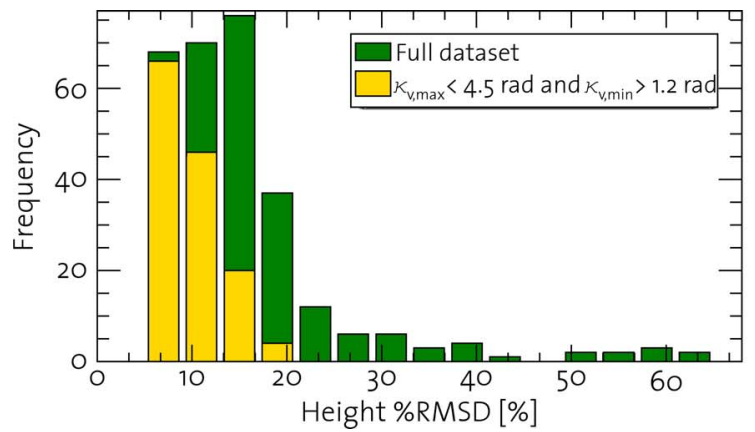

(a)

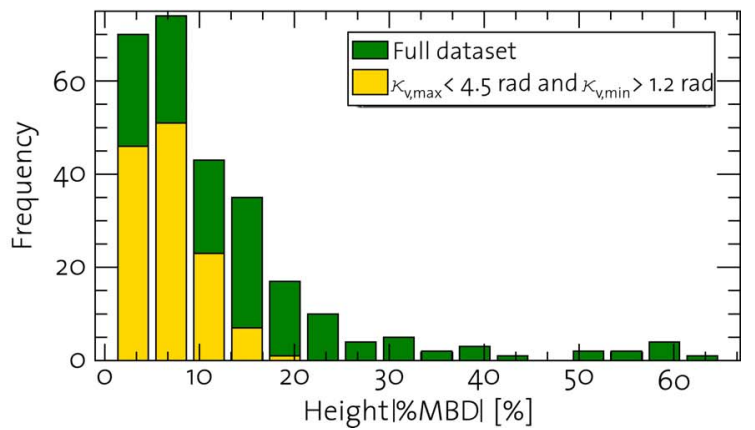

(b)

Fig. 7. Height (a) \%RMSD and (b) $|\% \mathrm{MBD}|$ in the dual-baseline case $\left(\gamma_{\mathrm{dec}, 1}=\gamma_{\mathrm{dec}, 2}=0.95\right)$ : the histogram of the full data set is in green; the histogram of the realizations whose $\kappa_{v, \max }<4.5 \mathrm{rad}$ and $\kappa_{v, \min }>1.2 \mathrm{rad}$ is in yellow.

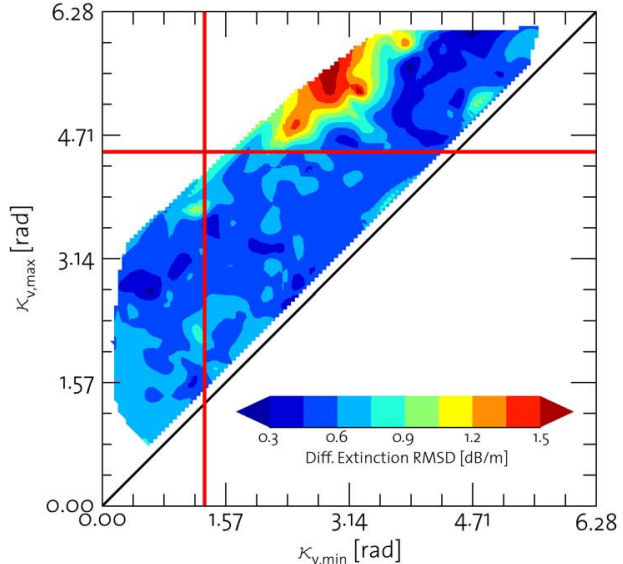

(a)

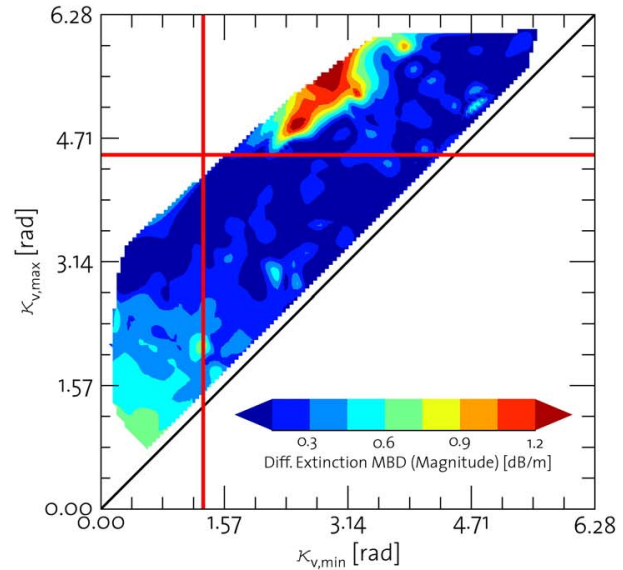

(b)

Fig. 8. Differential extinction (a) RMSD and (b) $|\mathrm{MBD}|$ in the dual-baseline case, with $\gamma_{\mathrm{dec}, 1}=\gamma_{\mathrm{dec}, 2}=0.95$ : the two axes refer to the realizations' minimum and maximum $\kappa_{v}$ (in rad). Two red thresholds are set at $\kappa_{v, \max }=4.5 \mathrm{rad}$ and $\kappa_{v, \min }=1.2 \mathrm{rad}$.

As in the case for $\kappa_{v, \text { max }}$, one can set a boundary for $\kappa_{v, \text { min }}$. If the threshold $\kappa_{v, \text { min }}>1.2 \mathrm{rad}$ is chosen, then the height is estimated with $18 \% \%$ RMSD accuracy for approximately $95 \%$ of the samples whose $\kappa_{v} \in[1.2,4.5] \mathrm{rad}$.

The results gained so far indicate the existence of a range of $\kappa_{v}$ values for which the estimation error due to either small or large $\kappa_{z}$ is minimized, and the inversion scheme is robust against nonvolumetric coherence losses. This finding is in agreement with a previous study in [16], conducted for the single-baseline case. For small $\kappa_{z}$, the reduced sensitivity of the OVoG complex coherence to the vegetation height explains the drop in accuracy as soon as an additional decorrelation 


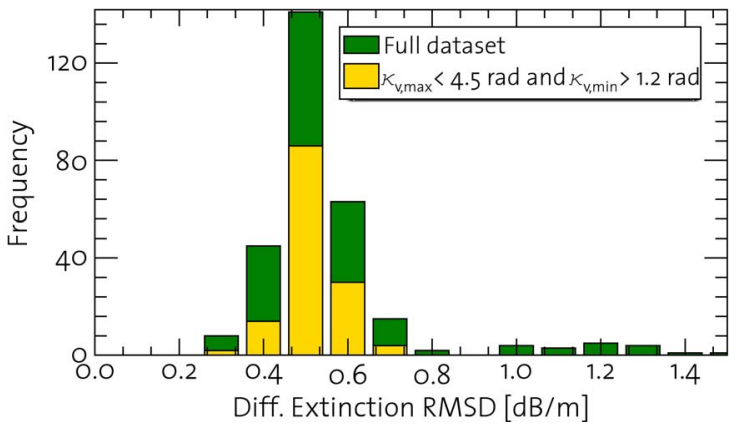

(a)

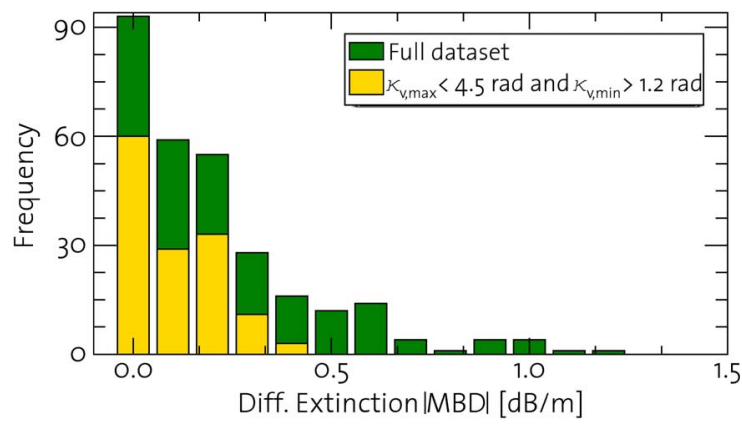

(b)

Fig. 9. Differential extinction (a) RMSD and (b) $|\mathrm{MBD}|$ in the dual-baseline case $\left(\gamma_{\mathrm{dec}, 1}=\gamma_{\mathrm{dec}, 2}=0.95\right)$ : the histogram of the full data set is in green; the histogram of the realizations whose $\kappa_{v, \max }<4.5 \mathrm{rad}$ and $\kappa_{v, \min }>$ $1.2 \mathrm{rad}$ is in yellow.

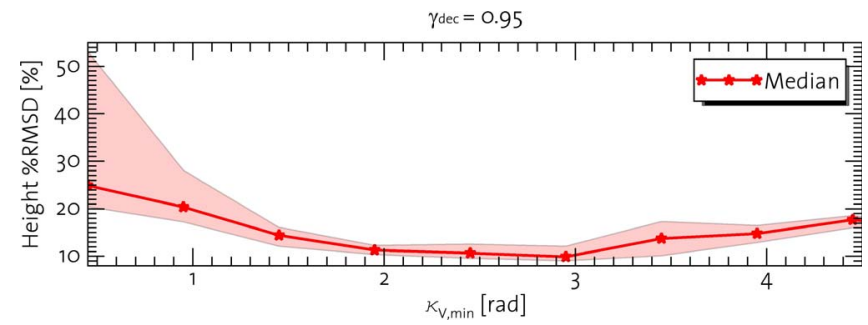

(a)

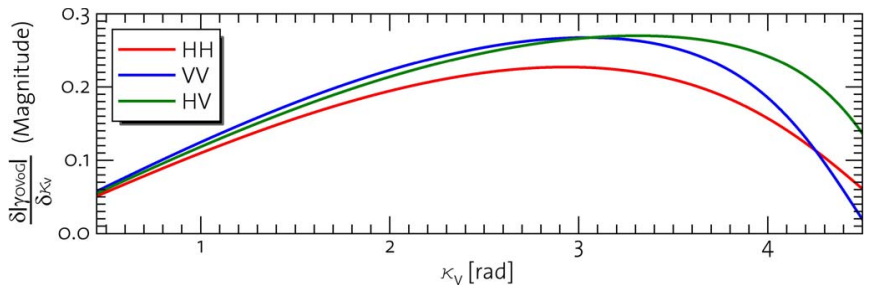

(b)

Fig. 10. (a) Height $\%$ RMSD distribution as a function of $\kappa_{v \text {,min }}$ (the solid line is the median, whereas the red region is the IQR), when $\gamma_{\mathrm{dec}, 1}=\gamma_{\mathrm{dec}, 2}=$ 0.95. (b) $\left|\frac{\partial\left|\gamma_{\mathrm{OVoG}}\right|}{\partial \kappa_{\mathrm{v}}}\right|$, for the three polarimetric channels: $\mathrm{HH}$ (red), VV (blue), and HV (green).

source is introduced. On the other hand, the performance degradation observed for large $\kappa_{z}$ is due to the increased volume decorrelation, which leads to an increased phase variation.

\section{B. Impact of the Number of Baselines}

1) Maize Scenario: Here, the Monte Carlo analysis for the maize scenario in Section IV-A is repeated for different $N b$, to investigate the effect of the number of spatial baselines on the

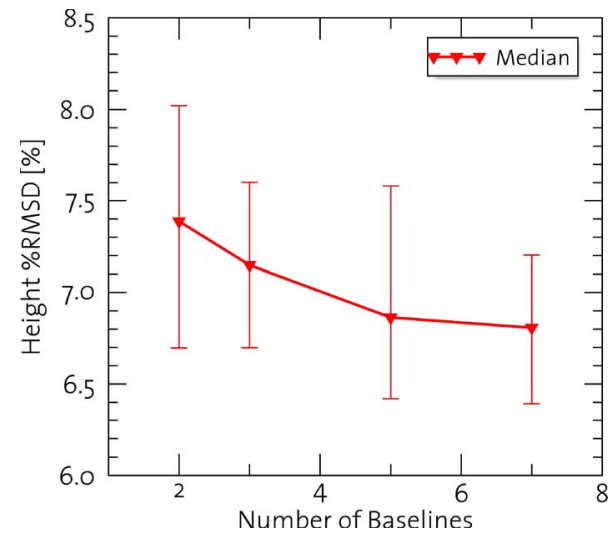

(a)

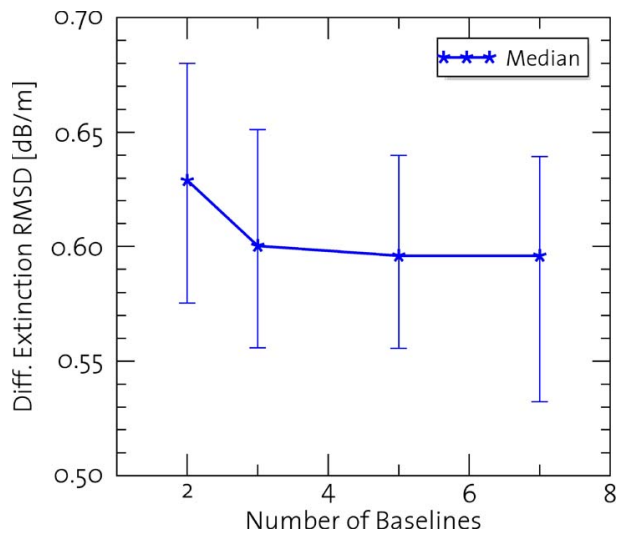

(b)

Fig. 11. Maize scenario. Distributions of the (a) height \%RMSD and the (b) differential extinction RMSD as a function of the number of baselines $N b$ : the solid line is the median, whereas the two whiskers refer to the lower and upper quartiles.

inversion performance. The inversion scheme is applied to a set of 100 realizations of the maize scenario. For each of them, $N b$ spatial baselines are randomly chosen such that $\kappa_{v, i} \in$ $[1.2,4.5] \mathrm{rad}$ and $\Delta \kappa_{v, i j}=\left|\kappa_{v, i}-\kappa_{v, j}\right|>0.2 \mathrm{rad}, \forall i, j=$ $1, \ldots, N b, i \neq j$.

The distribution of the height \%RMSD and that of the differential extinction RMSD are shown as a function of the number of baselines in Fig. 11(a) and (b), respectively. In both cases, the median of the distributions shows a decreasing trend with $N b$, implying a negative correlation between the estimation error and the number of available baselines. When compared with the dual-baseline case, the median of the height $\%$ RMSD distribution shows a reduction (by about 7\%) if $N b=5$ baselines are employed, whereas that of the differential extinction is reduced by $4.5 \%$ when $N b=3$. For this particular scenario, a further increase in the number of baselines does not substantially reduce the estimation error.

2) Any-Crop Scenario: A Monte Carlo analysis is carried out for the any-crop data set, to assess the impact of the number of baselines for a broad range of agricultural scenarios. Initially, 500 realizations of the any-crop scenario are generated for $N b=2$ and $\Delta z=0.4 \mathrm{~m}$, by fixing $\kappa_{v, 1}=1.2 \mathrm{rad}$ and $\kappa_{v, 2}=$ $2.8 \mathrm{rad}$. Subsequently, the same analysis is repeated for a higher number of baselines $(N b=3$ and $N b=5)$. In this case, the additional $\kappa_{v}$ values are selected within the range [1.2,2.8] rad (see Table IV). 
TABLE IV

Selected $\kappa_{v}$ For the Monte CARlo Analysis

\begin{tabular}{cc}
\hline Number of Baselines & $\kappa_{v}[\mathrm{rad}]$ \\
\hline \hline 2 & {$[1.2,2.8]$} \\
3 & {$[1.2,2,2.8]$} \\
5 & {$[1.2,1.6,2,2.4,2.8]$}
\end{tabular}

TABLE V

UPPER QUARTILE OF THE HEIGHT \%RMSD AND $\mid \%$ MBD DISTRIBUTIONS FOR THREE DIFFERENT $N b$, WHEN $\Delta z=0.4 \mathrm{~m}$

\begin{tabular}{lccc}
\hline \multicolumn{4}{c}{ Vegetation Height } \\
\hline & $N b=2$ & $N b=3$ & $N b=5$ \\
\hline \%MSD $_{75}[\%]$ & 7.8 & 7.3 & 6.7 \\
$\mid \%$ MBD $_{75}[\%]$ & 3.8 & 3.9 & 3.8
\end{tabular}

TABLE VI

UPPER QUARTILE OF THE DIFFERENTIAL EXTINCTION RMSD AND $|\mathrm{MBD}|$ DISTRIBUTIONS (IN $\mathrm{dB} / \mathrm{m}$ ) FOR THREE DIFFERENT $N b$, WHEN $\Delta z=0.4 \mathrm{~m}$

\begin{tabular}{lccc}
\hline \multicolumn{4}{c}{ Differential Extinction } \\
\hline \hline & $N b=2$ & $N b=3$ & $N b=5$ \\
\hline RMSD $_{75}[\mathrm{~dB} / \mathrm{m}]$ & 1.1 & 0.85 & 0.6 \\
$|\mathrm{MBD}|_{75}[\mathrm{~dB} / \mathrm{m}]$ & 0.23 & 0.25 & 0.25
\end{tabular}

As highlighted in Table $\mathrm{V}$, the 75th percentile of the height \%RMSD distribution varies between $7.8 \%$ in the dual-baseline case and $6.7 \%$ when $N b=5$, whereas no such dependence upon $\mathrm{Nb}$ is noticeable in the case of the height $|\% \mathrm{MBD}|$ (equal to $3.8 \%$ ). Interestingly, only a minor improvement in the variance of the estimates is found. Therefore, the accuracy of the estimated height does not change significantly whether one uses $N b>2$ baselines in the interval $\left[\kappa_{v, 1}, \kappa_{v, 2}\right]$ or two baselines whose $\kappa_{v}$ are the bounds of such an interval.

On the other hand, the results for the differential extinction show a pronounced reduction of the variance of the estimates as $N b$ increases (see Table VI). Compared with the dual-baseline case, the 75th percentile of its RMSD distribution improves by $45 \%$ (from 1.1 to $0.6 \mathrm{~dB} / \mathrm{m}$ ) when $N b=5$, whereas that of the $|\mathrm{MBD}|$ distribution is approximately $0.25 \mathrm{~dB} / \mathrm{m}$, independent of $N b$.

\section{Inversion Stability Over the Normalized Volume Power}

Here, the inversion scheme is applied to a given set of $N b=5$ spatial baselines whose $\kappa_{v}$ values range from $\kappa_{v, 1}=$ $1.2 \mathrm{rad}$ to $\kappa_{v, 5}=2.8 \mathrm{rad}$ and $\Delta \kappa_{v, i j}=\left|\kappa_{v, i}-\kappa_{v, j}\right|=0.4 \mathrm{rad}$, $\forall i, j=1, \ldots, N b, i \neq j$.

The aim of such an assessment is to investigate the sensitivity of the proposed algorithm to variations of the ground-to-volume scattering ratio. The analysis is carried out both for the maize and any-crop scenarios, by imposing a full-range of phase candidates and the constraint $\Delta z=0.4 \mathrm{~m}$.

The inversion stability is evaluated over the normalized volume backscattering power NVP, which is given by

$$
\mathrm{NVP}=\frac{P_{\mathrm{OV}}}{P_{S}+P_{\mathrm{OV}}}
$$

where $P_{\mathrm{OV}}=m_{v} \cdot \operatorname{tr}\left\{T_{\mathrm{OV}}\right\}$, and $P_{S}=m_{s} \cdot \operatorname{tr}\left\{T_{S}\right\}$. The operator $\operatorname{tr}\{A\}$ of a square matrix $A$ is the sum of the elements on its main diagonal. NVP is a real value ranging from 0 (no volume contribution) to 1 (pure volume contribution) and is

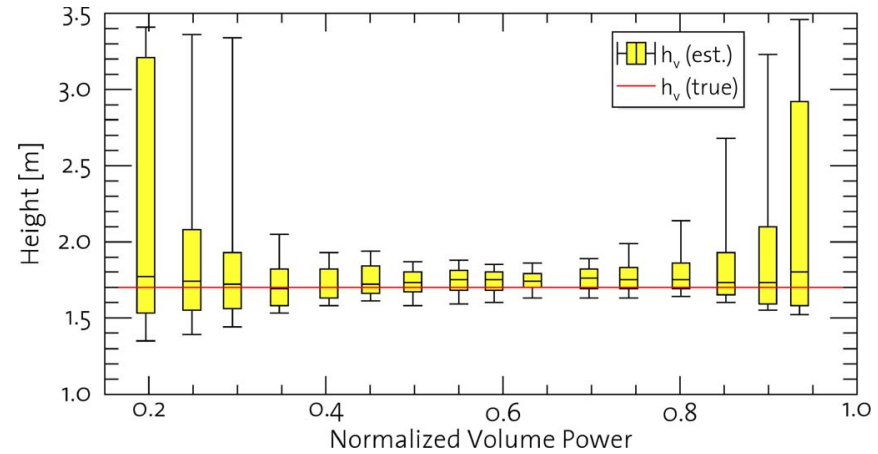

(a)

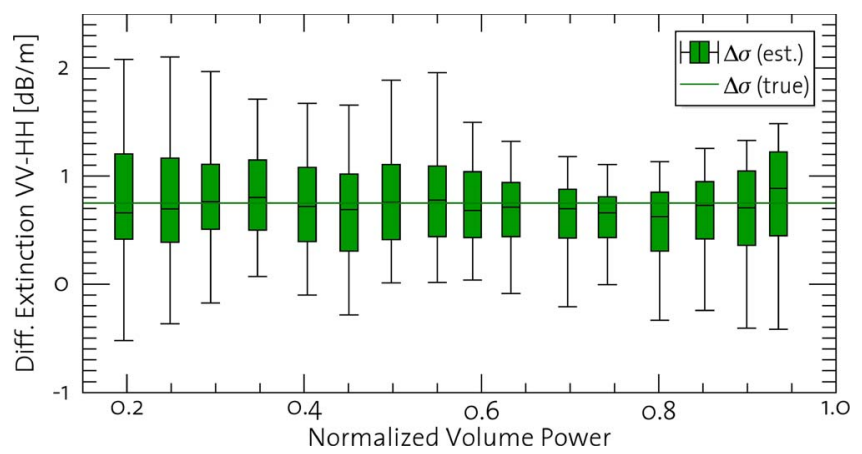

(b)

Fig. 12. (a) and (b) Box-and-whiskers plots as a function of the normalized volume power: the vegetation height $h_{V}$ is in yellow; the differential extinction $\Delta \sigma$ is in green. The solid lines refer to the simulated values.

inversely related to the ground-to-volume scattering ratio of the three polarimetric channels.

1) Maize Scenario, Full-Range: As a first approach, the $m_{s} / m_{v}$ ratio is varied, whereas all other OVoG parameters are kept fixed (see Table I).

Fig. 12 shows the box-and-whiskers plot (the two whiskers represent the 5th and 95th percentiles) of the estimated vegetation height $h_{V}$ and the differential extinction $\Delta \sigma$, whereas Tables VII and VIII summarize the RMSD and the MBD of the estimated ground-to-volume ratios and wave extinctions at three NVP values. The median of the estimates is consistent with the simulated values throughout the NVP range. On average, the mean bias for the vegetation height is well below $0.1 \mathrm{~m}$, whereas that of the differential extinction does not exceed $0.15 \mathrm{~dB} / \mathrm{m}$. Nevertheless, one may observe that the variability of the estimated $h_{V}$ is not constant over NVP: the height IQR increases as one moves toward low and high NVP values, and the 95th percentile tends to the upper bound of the solution space.

To partially explain the dependence of the estimates variability on NVP, one may have a closer look at the partial results of Step 3 of the inversion scheme (see Section III-C). Fig. 13 displays the $\sigma_{v v, 5}$ extinction candidates as a function of $\phi$, for three NVP values at the actual crop height. For the three scenarios, the partial derivative $\Delta_{v v, 5}=\left.\frac{\partial \sigma_{v v, 5}}{\partial \phi}\right|_{\phi_{5}}$ of $\sigma_{v v, 5}$ with respect to $\phi$ is evaluated at $\phi_{5}=\kappa_{z, 5} z_{0}$, where $z_{0}$ is the height of the ground.

The lowest absolute value of the derivative is found for $\mathrm{NVP}=0.6\left(\left|\Delta_{v v, 5}\right|=0.65\right)$. On the other hand, this measure tends to increase for low $\left(\left|\Delta_{v v, 5}\right|=16.3\right.$ at $\left.\mathrm{NVP}=0.3\right)$ and high $\left(\left|\Delta_{v v, 5}\right|=5\right.$ at $\left.\mathrm{NVP}=0.9\right) \mathrm{NVP}$ values, which means 
TABLE VII

InVERsion STABILITy Against THE NORMALIZED Volume BACKSCATTERING POWER: RMSD AND MBD of the Estimated Ground-TO-Volume Ratios at Three SElected NVP VALUES

\begin{tabular}{lccccccccc}
\hline & \multicolumn{3}{c}{ NVP $=0.3$} & \multicolumn{3}{c}{ NVP = 0.6 } & \multicolumn{3}{c}{ NVP $=0.9$} \\
\hline \hline & $\mu_{H H}$ & $\mu_{V V}$ & $\mu_{H V}$ & $\mu_{H H}$ & $\mu_{V V}$ & $\mu_{H V}$ & $\mu_{H H}$ & $\mu_{V V}$ & $\mu_{H V}$ \\
\hline True value & 2.50 & 2.352 & 1.598 & 0.725 & 0.682 & 0.463 & 0.117 & 0.11 & 0.075 \\
RMSD & 1.858 & 1.529 & 1.06 & 0.463 & 0.324 & 0.303 & 0.418 & 0.221 & 0.273 \\
MBD & -0.589 & -0.592 & -0.351 & 0.081 & 0.045 & 0.053 & 0.172 & 0.089 & 0.112 \\
\hline
\end{tabular}

TABLE VIII

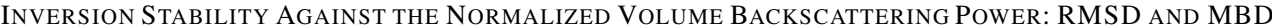

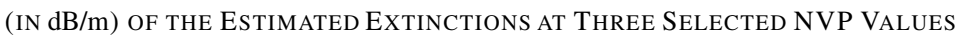

\begin{tabular}{lccccccccc}
\hline & \multicolumn{3}{c}{$\mathrm{NVP}=0.3$} & \multicolumn{3}{c}{$\mathrm{NVP}=0.6$} & \multicolumn{3}{c}{$\mathrm{NVP}=0.9$} \\
\hline \hline & $\sigma_{H H}$ & $\sigma_{V V}$ & $\sigma_{H V}$ & $\sigma_{H H}$ & $\sigma_{V V}$ & $\sigma_{H V}$ & $\sigma_{H H}$ & $\sigma_{V V}$ & $\sigma_{H V}$ \\
\hline True value $[\mathrm{dB} / \mathrm{m}]$ & 0.25 & 1 & 0.625 & 0.25 & 1 & 0.625 & 0.25 & 1 & 0.625 \\
RMSD $[\mathrm{dB} / \mathrm{m}]$ & 1.49 & 1.42 & 2.12 & 0.77 & 1.14 & 0.94 & 1.28 & 1.53 & 1.38 \\
MBD $[\mathrm{dB} / \mathrm{m}]$ & 0.93 & 0.76 & 0.53 & 0.23 & 0.16 & 0.15 & 0.21 & 0.11 & 0.14 \\
\hline
\end{tabular}

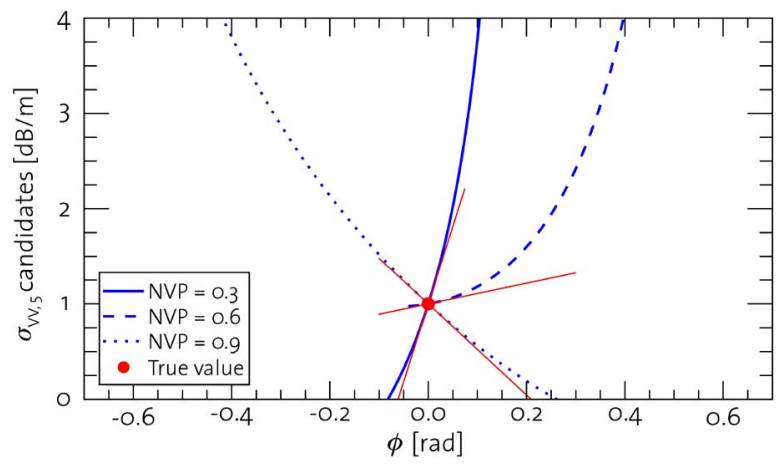

Fig. 13. Extinction candidates $\sigma_{v v, 5}$ as a function of $\phi\left(h_{V}=1.7 \mathrm{~m}\right)$, computed for (solid line) NVP $=0.3$, (dashed line) NVP $=0.6$, and (dotted line) NVP $=0.9$. For each scenario, a line is drawn whose slope is the partial derivative of $\sigma_{v v, 5}$ with respect to $\phi$ (evaluated at $\phi_{5}=\kappa_{z, 5} z_{0}$ ).

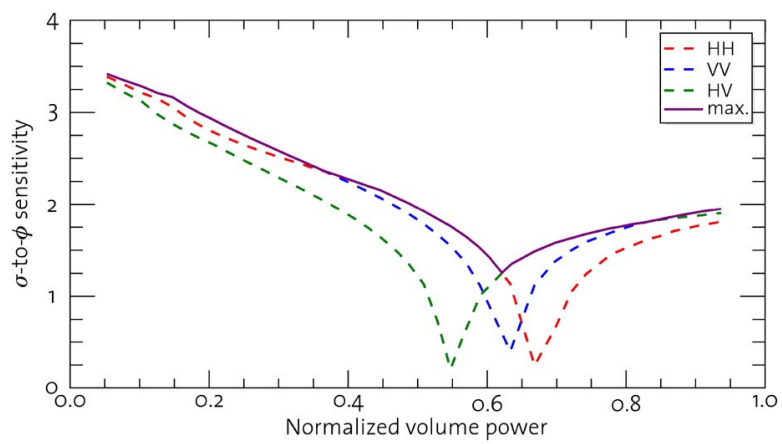

Fig. 14. Derivative $\Delta_{p q}$ as a function of the normalized volume power for (dashed red line) HH, (dashed blue line) VV, and (dashed green line) HV. The solid purple line shows the maximum value of such a derivative (among the three channels), as a function of NVP.

that a small variation of $\phi$ results in a significant change of $\sigma_{v v, 5}$ values, which possibly causes the performance degradation.

The derivative $\Delta_{p q}$ (the subscripts $p$ and $q$ refer to the receive and transmit polarizations, respectively) is now computed by averaging the contributions from the $N b$ baselines, i.e.,

$$
\Delta_{p q}=\ln \sqrt{\sum_{i=1}^{N b}\left(\left.\frac{\partial \sigma_{p q, i}}{\partial \phi}\right|_{\phi_{i}}\right)^{2}} .
$$

In Fig. 14, $\Delta_{p q}$ is displayed as a function of NVP for the three polarimetric channels. A conclusive interpretation of the relationship between the estimates variability and the curves in Fig. 14 is not straightforward, due to the concurrent impact of

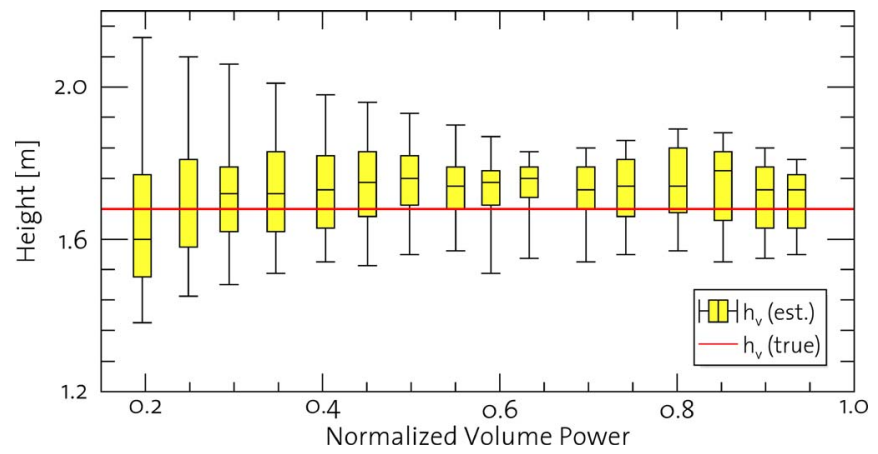

(a)

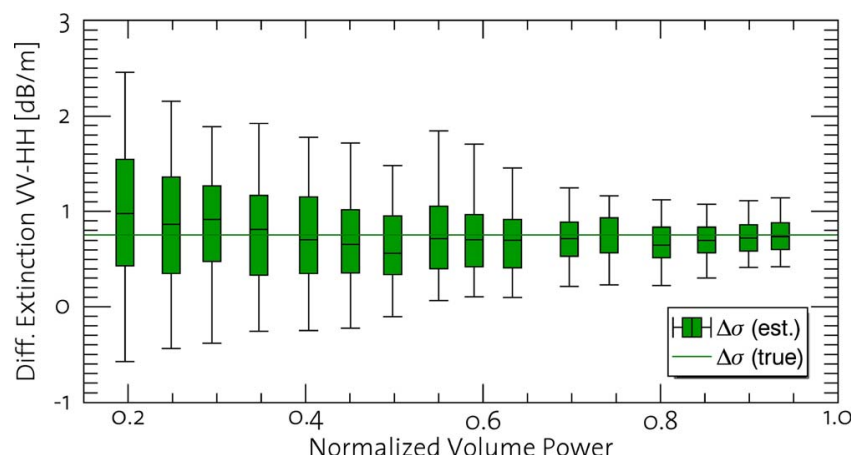

(b)

Fig. 15. (a) and (b) Box-and-whiskers plots as a function of the normalized volume power, when $\Delta z=0.4 \mathrm{~m}$ : the vegetation height $h_{V}$ is in yellow; the differential extinction $\Delta \sigma$ is in green. The solid lines refer to the simulated values.

the three polarimetric channels on the inversion results. Nevertheless, one can still observe a positive correlation between the IQR of the height estimates and the maximum $\Delta_{p q}$ (see the purple curve in Fig. 14) for NVP $<0.8$. Consequently, the sensitivity of $\sigma_{p q}$ to changes of the $\phi_{p q, i}$ values (due to volume decorrelation or other decorrelation sources) is likely to affect the robustness of the model inversion, as it will presumably play a strong role on the subsequent minimization over the $\mathrm{Nb}$ baselines of Step 3.

2) Maize Scenario, $\Delta z=0.4 \mathrm{~m}$ : A further assessment is carried out over the maize scenario on a restricted set of ground phase candidates. The results obtained for $\Delta z=0.4 \mathrm{~m}$ are presented in Fig. 15 and Tables IX and X. The variability of the estimated height is now evidently reduced, when compared with the full-range case (maximum height IQR below $0.3 \mathrm{~m}$ ), 
TABLE IX

INVERSiON STABILITY AgAinst THE NORMALIZED VOLUME BACKSCATTERING POWER: RMSD AND MBD of the Estimated Ground-to-Volume Ratios at Three Selected NVP Values, When $\Delta z=0.4 \mathrm{~m}$

\begin{tabular}{lccccccccc}
\hline & \multicolumn{3}{c}{ NVP $=0.3$} & \multicolumn{3}{c}{ NVP $=0.6$} & \multicolumn{3}{c}{ NVP $=0.9$} \\
\hline \hline & $\mu_{H H}$ & $\mu_{V V}$ & $\mu_{H V}$ & $\mu_{H H}$ & $\mu_{V V}$ & $\mu_{H V}$ & $\mu_{H H}$ & $\mu_{V V}$ & $\mu_{H V}$ \\
True value & 2.50 & 2.352 & 1.598 & 0.725 & 0.682 & 0.463 & 0.117 & 0.11 & 0.075 \\
RMSD & 1.511 & 1.08 & 0.887 & 0.406 & 0.275 & 0.265 & 0.128 & 0.088 & 0.093 \\
MBD & 0.447 & 0.353 & 0.317 & 0.139 & 0.086 & 0.067 & 0.028 & 0.014 & 0.02
\end{tabular}

TABLE X

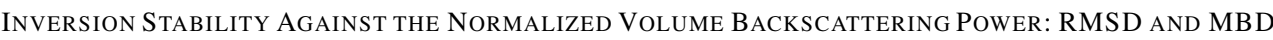
(IN dB/m) Of the Estimated Extinctions at Three Selected NVP Values, When $\Delta z=0.4 \mathrm{~m}$

\begin{tabular}{llcccccccccc}
\hline & \multicolumn{3}{c}{ NVP $=0.3$} & \multicolumn{3}{c}{ NVP = 0.6 } & \multicolumn{3}{c}{ NVP = 0.9 } \\
\hline \hline True value [dB/m] & $\sigma_{H H}$ & $\sigma_{V V}$ & $\sigma_{H V}$ & $\sigma_{H H}$ & $\sigma_{V V}$ & $\sigma_{H V}$ & $\sigma_{H H}$ & $\sigma_{V V}$ & $\sigma_{H V}$ \\
RMSD [dB/m] & 0.60 & 1 & 0.625 & 0.25 & 1 & 0.625 & 0.25 & 1 & 0.625 \\
MBD [dB/m] & 0.23 & 0.32 & 0.68 & 0.92 & 1.37 & 1.14 & 0.51 & 0.7 & 0.59 \\
\hline
\end{tabular}

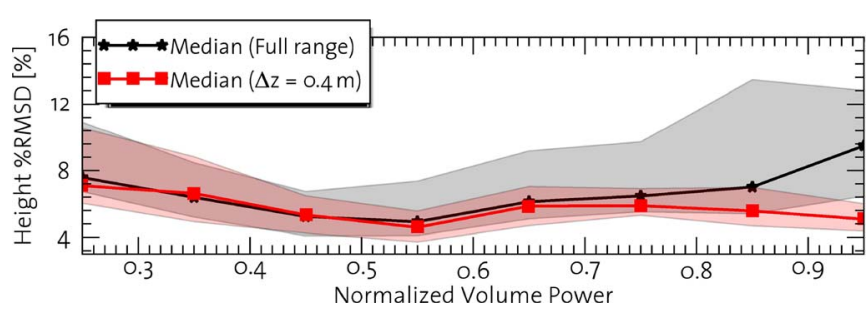

(a)

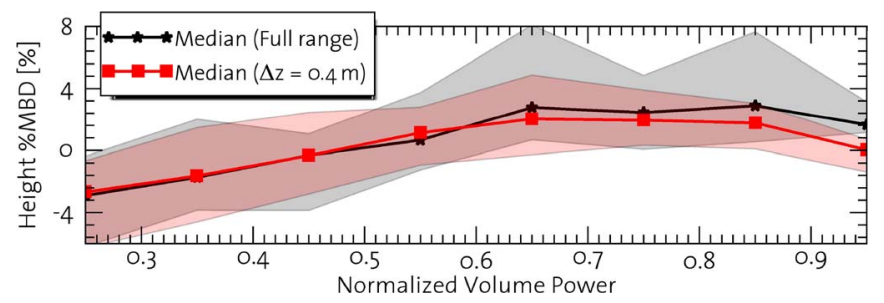

(b)

Fig. 16. Height (a) \%RMSD and (b) \%MBD distributions as a function of the normalized volume power for the (black stars) full $\phi$-range case and (red squares) $\Delta z=0.4 \mathrm{~m}$ : the solid lines refer to the median, whereas the colored region is the IQR.

although weak dependence on NVP is still noticeable. Interestingly, the IQR of the differential extinction is decreasing with NVP, and it ranges between 1.2 and $0.3 \mathrm{~dB} / \mathrm{m}$. We believe that such a variability of the retrieved $\Delta \sigma$ may be related to the trend of the derivative $\left.\frac{\partial \angle \gamma_{\mathrm{OVoG}, i}}{\partial \sigma}\right|_{\sigma_{p q}}$, which is monotonically increasing with NVP. As a result, the sensitivity of the estimates to variations of the measured coherences is stronger at lower NVP values, thus resulting in a higher estimation error.

3) Any-Crop Scenario: A further validation is now performed over 500 realizations of the any-crop scenario, to assess the consistency of the previous results for different agricultural scenarios.

Here, the results of the full-range case are compared with those obtained by imposing $\Delta z=0.4 \mathrm{~m}$.

The distributions of the height $\% \mathrm{MBD}$ and $\% \mathrm{RMSD}$, as a function of NVP, are shown in Fig. 16; those of the differential extinction's MBD and RMSD are shown in Fig. 17. As highlighted in Fig. 16, an overall degradation of the height inversion performance is noticeable when no boundary on $\phi$ is imposed. In the full-range case, it is reasonable to assume a higher variability of the estimates as one moves toward high NVP values: when $0.55<\mathrm{NVP}<0.95$, the median of the $\%$ RMSD

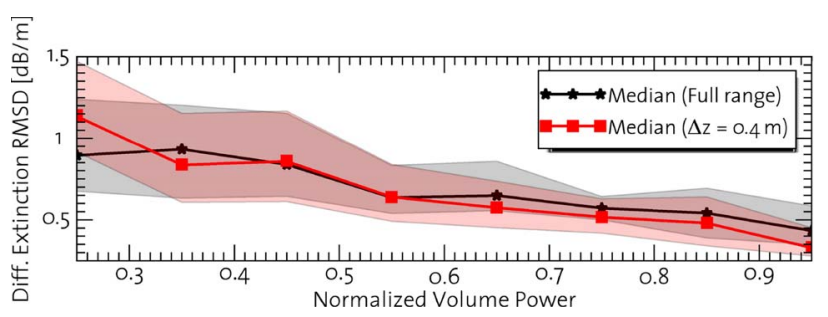

(a)

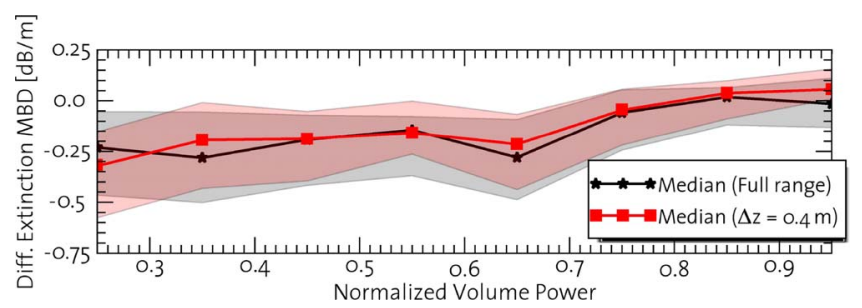

(b)

Fig. 17. Differential extinction (a) RMSD and (b) MBD distributions (in $\mathrm{dB} / \mathrm{m}$ ) as a function of the normalized volume power for the (black stars) full $\phi$-range case and (red squares) $\Delta z=0.4 \mathrm{~m}$ : the solid lines refer to the median, whereas the colored region is the IQR.

\section{TABLE XI}

INVERSION STABILITY AGAINST THE NORMALIZED VOLUME BACKSCATTERING POWER: FRACTION OF KEPT REALIZATIONS (IN \%) FOR THE FULL-RANGE AND $\Delta z=0.4 \mathrm{~m}$ CASES

\begin{tabular}{lcc}
\hline \multicolumn{3}{c}{ Fraction of kept realisations [\%] } \\
\hline \hline & full-range & $\Delta z=0.4 \mathrm{~m}$ \\
\hline $\mathrm{NVP}=0.3$ & 52 & 40 \\
$\mathrm{NVP}=0.45$ & 76 & 75 \\
$\mathrm{NVP}=0.55$ & 95 & 93 \\
$\mathrm{NVP}=0.7$ & 100 & 100 \\
$\mathrm{NVP}=0.85$ & 100 & 100 \\
\hline
\end{tabular}

distribution is monotonically increasing with NVP, whereas no such trend is found for that of the \%MBD distribution (equal to $2 \%$ ). On the other hand, if $\Delta z=0.4 \mathrm{~m}$, then the median and the IQR of the \%RMSD distribution are independent of NVP over the same interval (median of $6 \%$ and IQR $=2.5 \%$ ), whereas a slight degradation is still noticeable for low NVP values (e.g., median of $7 \%$ and IQR $=6 \%$ at $\mathrm{NVP}=0.25$ ) The differential extinction RMSD and MBD distributions are shown in Fig. 17.

In Table XI, the fraction of realizations for which the height $\%$ MBD and \% RMSD have been computed (i.e., where a valid solution exists for at least $75 \%$ of the samples) is reported against the values of NVP. Based on these simulated data, it 
TABLE XII

HEIGHT INVERSION STATISTICS FOR DIFFERENT NUMBERS OF INDEPENDENT LOOKS

\begin{tabular}{ccccccc}
\hline $\begin{array}{c}\text { Number } \\
\text { of Looks }\end{array}$ & \multicolumn{2}{c}{ NVP $=0.2$} & \multicolumn{2}{c}{ NVP $=0.6$} & \multicolumn{2}{c}{ NVP $=0.8$} \\
\hline \hline & \%MBD & \%RMSD & \%MBD & \%RMSD & \%MBD & \%RMSD \\
\hline 81 & -8.5 & 21.7 & 2.9 & 7.2 & 2.0 & 8.5 \\
121 & -4.5 & 19.8 & 2.7 & 6.8 & 2.2 & 8.1 \\
289 & -1.0 & 15.9 & 1.8 & 6.0 & 1.9 & 7.8 \\
441 & 0.54 & 15.0 & 1.3 & 5.7 & 1.2 & 7.1 \\
729 & -0.7 & 12.3 & -1.1 & 5.6 & 0.8 & 6.1 \\
\hline
\end{tabular}

seems that this fraction, which is typically higher in the fullrange case, tends to increase with NVP, being above $90 \%$ if $0.55 \leq \mathrm{NVP} \leq 0.85$. When $\mathrm{NVP} \leq 0.45$, the algorithm does not provide a valid solution for a larger number (i.e., up to $60 \%$ ) of simulated realizations. In this case, the failure case described in Section III-C is often observed for all elements of the $\left(h_{v}, \sigma\right)$ search space, presumably due to the very high sensitivity of the extinction values to variations of the ground phase (as already discussed for the maize scenario and shown in Fig. 13).

\section{Impact of the Number of Looks}

The vegetation height is now estimated using different numbers of independent looks $L$. The assessment is performed over the maize scenario, by imposing $\Delta z=0.4 \mathrm{~m}$. The spatial baselines employed for the inversion are the same as those in Section IV-C.

Table XII presents the \%MBD and the \%RMSD of the retrieved height against $L$ for three different values of NVP. The results are according to the expectations: both statistics, in magnitude, tend to decrease with the increasing number of looks. As an example, reducing the number of looks by a factor of 9 (from 729 to 81 ) causes the height's \%RMSD to increase by $22 \%-43 \%$.

When $L \geq 289$, the results show that the magnitude of the $\% \mathrm{MBD}$ is always below $2 \%$ and it does not substantially change with NVP. For a lower number of looks, one may observe that the height is considerably underestimated when NVP = 0.2 , where a pronounced sensitivity of the model parameters to variations of the measurements was already found (see Section IV-C).

\section{EXPERIMENTAL DATA ANALYSIS}

\section{A. Test Site and Data Presentation}

Fully polarimetric repeat-pass interferometric SAR data are acquired over an agricultural area in the proximity of Wallerfing, Germany (48.684 N, 12.88 E) in July 2013 by the experimental F-SAR, an airborne radar system developed and operated by the DLR.

The scene extends over an area of approximately $3.5 \times$ $6 \mathrm{~km}^{2}$. The topography is rather flat; the difference between the maximum and minimum altitudes is about $95 \mathrm{~m}$.

In situ measurements have been simultaneously collected for four different crop types (maize, barley, wheat, and rape) over six agricultural fields. The measurements have been performed at two separate locations within each field. The averaged values of the crop height $h_{V}$, as well as the plant phenological stage
TABLE XIII

Ground Measurements From the Test Site of Wallerfing, GERMANY: MEAN VEGETATION HEIGHT $h_{V}(\mathrm{~m})$, Volumetric WATER Content (VWC, \%), and Plant Phenological Stage BBCH

\begin{tabular}{lcccc}
\hline ID & Type & Mean $h_{V}[\mathrm{~m}]$ & VWC [\%] & BBCH \\
\hline \hline W1 & Wheat & 0.65 & 55 & $71-73$ \\
W4 & Wheat & 0.80 & 57 & $77-83$ \\
B3 & Barley & 0.75 & 18 & $89-92$ \\
C3 & Maize & 1.60 & 82 & 36 \\
C4 & Maize & 1.75 & 84 & $39-51$ \\
R & Rape & 1.40 & 66 & $88-89$ \\
\hline
\end{tabular}

Biologische Bundesanstalt, Bundessortenamt und CHemische Industrie $(\mathrm{BBCH})$ and the volumetric water content (VWC), are summarized in Table XIII.

Fig. 18 shows the X-band Pauli RGB image of the test site. Note that the radar measurements have been calibrated so that the polarimetric coherency matrix represents the radar brightness $\beta^{0}$. In Fig. 18, the crop fields employed in the study are highlighted with solid yellow lines. Some photographs of these fields are shown in Fig. 19.

Multibaseline acquisitions are available: four tracks at L-band and seven tracks at C- and X-bands, with a temporal baseline of about $10 \mathrm{~min}$ between consecutive passes. The spatial baselines at L-band range from 25 to $115 \mathrm{~m}$, corresponding to $\kappa_{z}$ values, in near range, between 1 and $4.5 \mathrm{~m}^{-1}$. At $\mathrm{C}$ - and X-bands, the spatial baselines range from 5 to $40 \mathrm{~m}$, corresponding to $\kappa_{z}$ values, in near range, between 0.8 and $6.2 \mathrm{~m}^{-1}$ and between 1.5 and $11.5 \mathrm{~m}^{-1}$, respectively.

For the experimental assessment, six different baseline configurations for each of the three bands are used. The set of $\kappa_{v}$ values associated with each crop is summarized in Fig. 20. Note that the lower and upper bounds of such sets show remarkable dependence on the sensor frequency.

The bandwidth at L-band is $150 \mathrm{MHz}$, thus giving a slantrange resolution of about $1 \mathrm{~m}$, whereas it is equal to $384 \mathrm{MHz}$ at $\mathrm{C}$ - and X-bands (slant-range resolution of $0.39 \mathrm{~m}$ ). The altitude of the sensor is $3.1 \mathrm{~km}$ above ground, and the look angle goes from $25^{\circ}$ in near range to $60^{\circ}$ in far range.

To reduce the impact of geometric decorrelation, range spectral filtering is employed over the F-SAR acquisitions [26]. In addition, a $15 \times 15$ boxcar averaging of the data is applied, which gives an equivalent number of looks of about 66 and 80 at L-band and C-/X-band, respectively.

Some a priori optimization constraints are used in order to avoid local minima and to enhance the speed of the inversion algorithm: the maximum vegetation height is here set to the height of ambiguity of the smallest baseline, whereas the maximum extinction coefficient is equal to $4.5 \mathrm{~dB} / \mathrm{m}$. Furthermore, the search space for the ground phase is constrained to an interval, by using the following methodology.

- For each baseline, a first-guess estimate of the ground phase is computed by means of the RVoG ML line fit procedure [25] and then compensated for.

- The range of ground phase values $\phi_{p q, i}$ is then reduced to a neighborhood $\left[-\kappa_{z, i} \frac{\Delta z}{2}, \kappa_{z, i} \frac{\Delta z}{2}\right]$, where $\Delta z=1 \mathrm{~m}$, and $\kappa_{z, i} \frac{\Delta z}{2}<\pi$.

For the sake of simplicity, the eigenpolarization basis is again identified with the $H-V$ linear basis. 


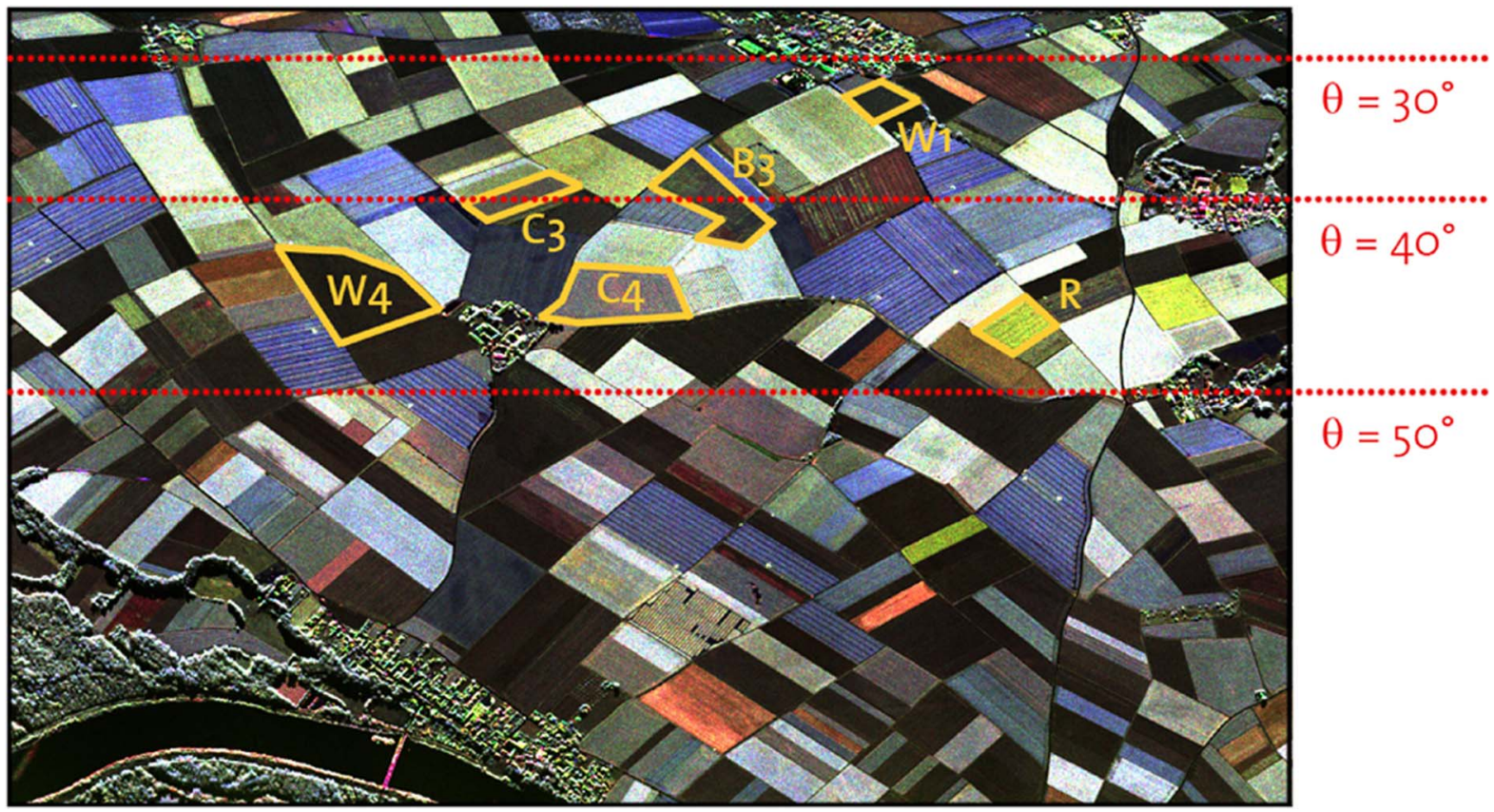

Fig. 18. Test site of Wallerfing, Germany: X-band Pauli RGB image $\left(\theta\right.$ is the radar incidence angle). Radar brightness $\beta^{0}:-30 \mathrm{~dB}<\beta_{\mathrm{HH}}^{0}<32 \mathrm{~dB}$, $-30 \mathrm{~dB}<\beta_{\mathrm{VV}}^{0}<32 \mathrm{~dB}$, and $-37 \mathrm{~dB}<\beta_{\mathrm{HV}}^{0}<20 \mathrm{~dB}$.

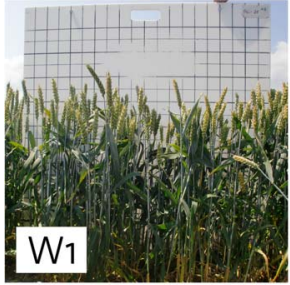

(a)

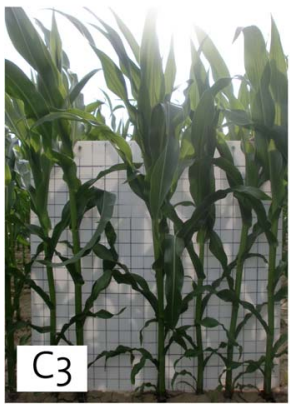

(d)

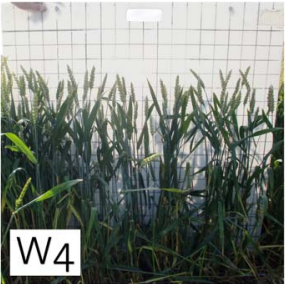

(b)

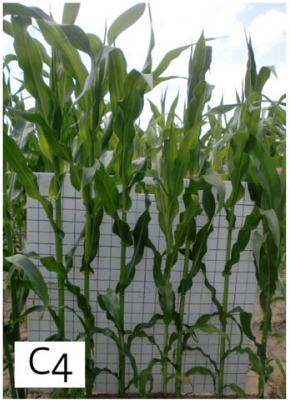

(e)

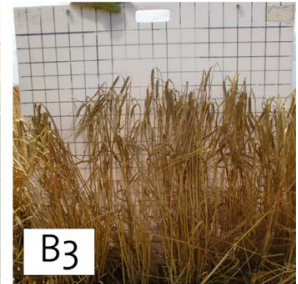

(c)

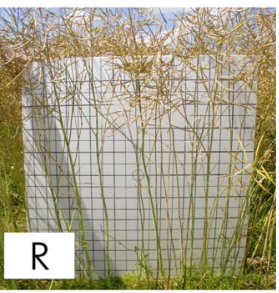

(f)

Fig. 19. Photographs of the case studies at the acquisition date.

\section{B. Dual-Baseline Case: Impact of $\kappa_{z}$ on the Inversion Results}

The proposed algorithm is applied to all possible dualbaseline configurations, and the performance is evaluated with respect to the vegetation height. The goal is to evaluate how the estimation results are influenced by $\kappa_{z}$ and to relate each crop to its optimal dual-baseline configuration among the available combinations.

In Fig. 21, the estimated height of the maize field $\mathrm{C} 4$ at L-band is displayed as a function of $\kappa_{v \text {,max }}$ (i.e., the largest $\kappa_{z}$ ). In Fig. 22, the same statistics are shown for the barley field B3 at X-band. Note that these trends are computed for different values of $\kappa_{v \text {,min }}$. The solid line refers to the median, whereas the whiskers represent the lower and upper quartiles.
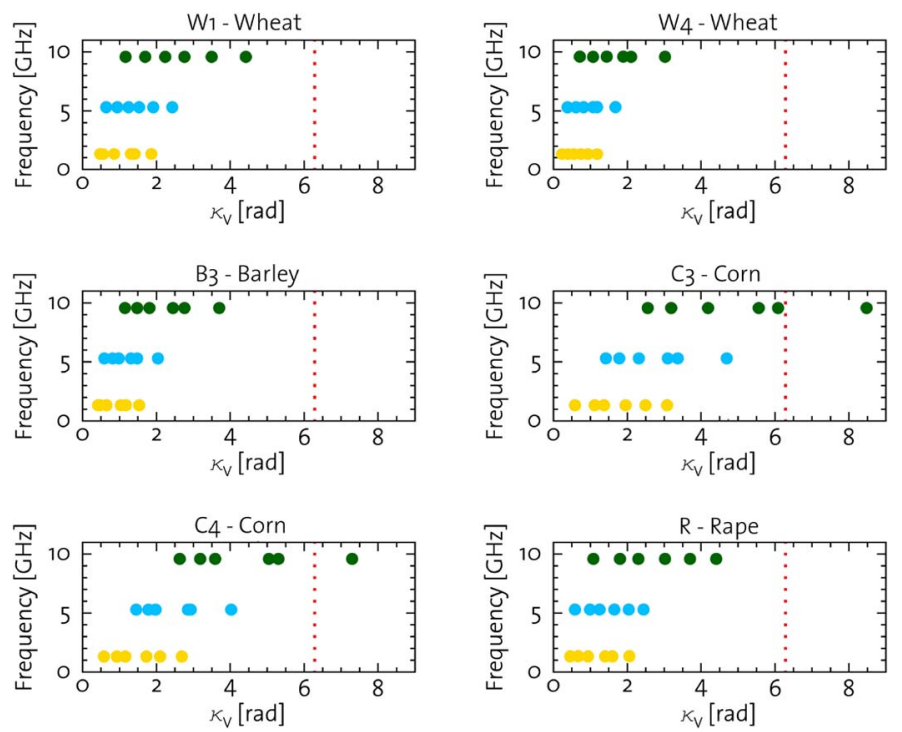

Fig. 20. Test site of Wallerfing, Germany: list of the available $\kappa_{v}$ at L-band (yellow), C-band (light blue), and X-band (green). The dashed red line is plotted for $\kappa_{v}=2 \pi$ (i.e., the mean height is equal to the height of ambiguity).

As suggested by the decreasing trend of the height bias with $\kappa_{v \text {,max }}$ which was found for both fields, the height estimates seem to suffer from a more severe overestimation when smaller $\kappa_{z}$ are employed, due to the poorer sensitivity of the Pol-InSAR coherence to height variations. This finding is in agreement with theoretical [16] and experimental investigations [10] and consistent with the assessment in Section IV. As an example, the height of the maize field, which is consistent with the ground measurements if $\kappa_{v, \max }>2.1 \mathrm{rad}$, is significantly overestimated (i.e., by $37 \%$ ) when the two smallest $\kappa_{z}$ are used (i.e., $\kappa_{v, 1}=0.58 \mathrm{rad}$ and $\left.\kappa_{v, 2}=0.93 \mathrm{rad}\right)$. Furthermore, the height bias of the barley field ranges from $24 \%$ when $\kappa_{v \text {,min }}=1.1 \mathrm{rad}$ and $\kappa_{v, \text { max }}=1.7 \mathrm{rad}$ to $10 \%$ when $\kappa_{v, \text { max }}=3.7 \mathrm{rad}$. 


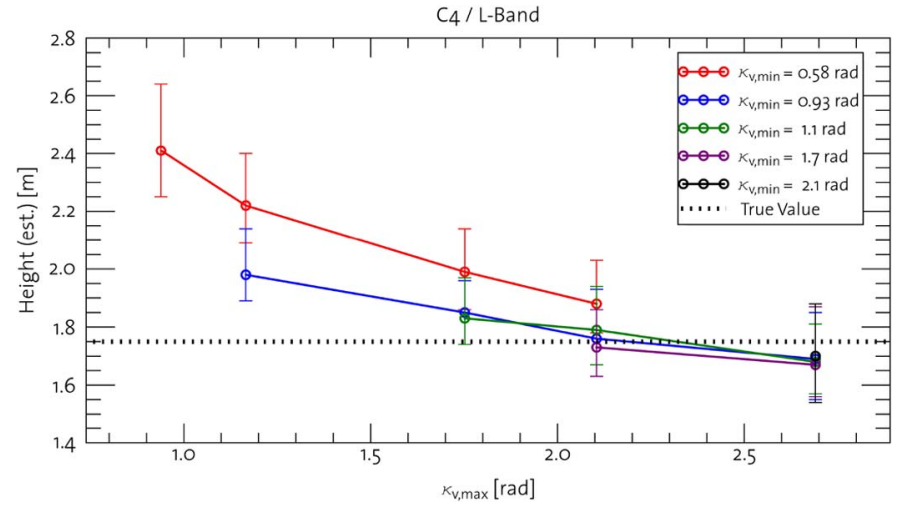

Fig. 21. Experimental data analysis: height inversion results for maize $\mathrm{C} 4$ at L-band.

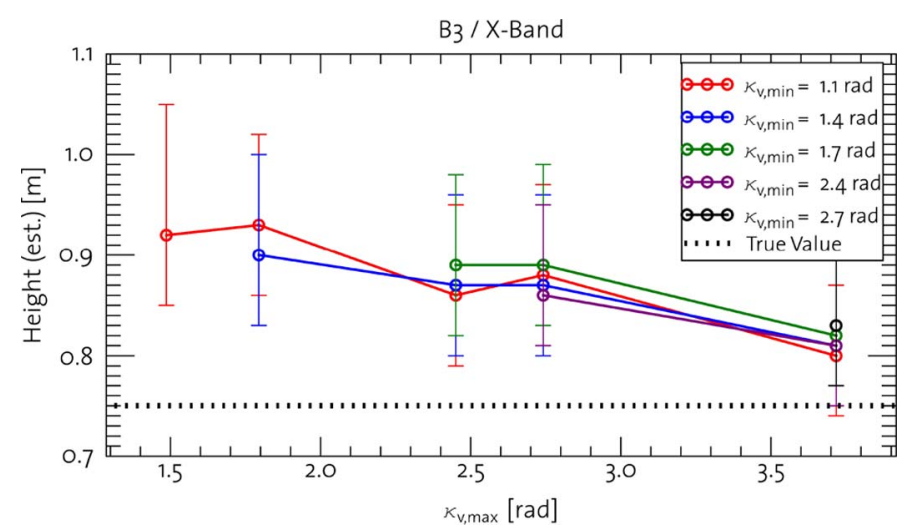

Fig. 22. Experimental data analysis: height inversion results for barley B3 at X-band.

Unfortunately, no conclusions about the inversion performance for large $\kappa_{z}$ can be drawn for the selected scenarios, as the maximum available $\kappa_{v}$ values do not exceed 2.7 and $3.7 \mathrm{rad}$ for maize and barley, respectively.

The same analysis is repeated for the other fields of the Wallerfing test site, at all frequencies. In Fig. 23, each crop is related to its best dual-baseline configuration, according to the $\%$ RMSD of the vegetation height. On average, the associated $\kappa_{v}$ values are located within the $\kappa_{v}$ range in Section IV-A. With regard to $\mathrm{W} 4$, the selected $\kappa_{v \text {,min }}$ at $\mathrm{L}$ - and C-bands is below the lower bound of this $\kappa_{v}$ range ( $\kappa_{v \text {,min }}$ is equal to 0.94 and $1.18 \mathrm{rad}$, respectively), as all the available $\kappa_{v}$ values are very small (see Fig. 20).

\section{Dual-Baseline Case: Estimation of Height and Differential Extinction}

Here, the inversion results obtained with the configurations in Fig. 23 are reported in detail.

Fig. 24 shows the box-and-whiskers plot of the estimated height as a function of the frequency for each of the six fields. In Fig. 25, the estimated height is represented in the slant-range/ azimuth geometry.

For the maize fields $\mathrm{C} 3$ and $\mathrm{C} 4$, the estimated height is robust against the frequency and characterized by a small bias (about $18 \%$ in the worst case), which is in agreement with the results, as shown in [12]. Note that the height is slightly

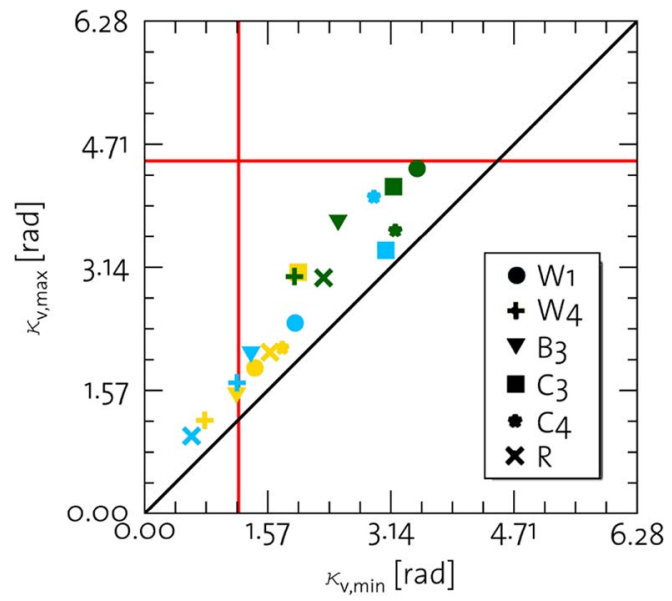

Fig. 23. Test site of Wallerfing, Germany: best $\kappa_{v}$ configurations in the dualbaseline case. The symbol refers to the crop type, whereas the color is related to the sensor frequency: L-band (yellow), C-band (light blue), and X-band (green). The red vertical line is drawn at $\kappa_{v, \text { min }}=1.2 \mathrm{rad}$ (i.e., the lower bound of the $\kappa_{v}$ range in Section IV-A), whereas the red horizontal line is drawn at $\kappa_{v, \max }=4.5 \mathrm{rad}$ (i.e., the upper bound of the $\kappa_{v}$ range in Section IV-A).

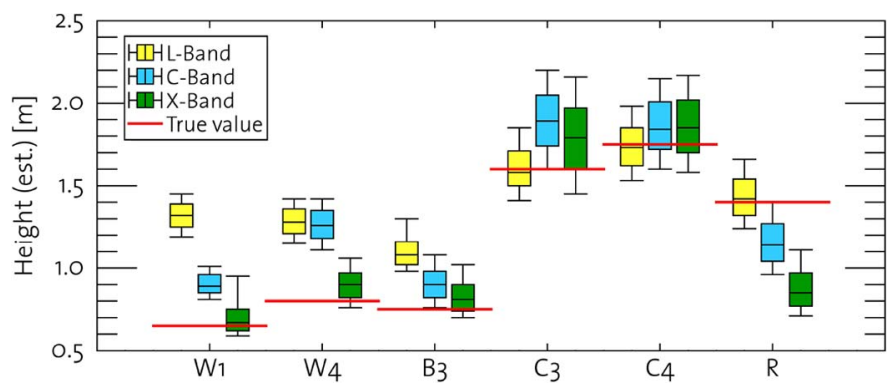

Fig. 24. Experimental data analysis: estimated vegetation height $h_{V}$ (dualbaseline case) at L-band (yellow), C-band (light blue), and X-band (green). The red solid line refers to the in situ measured height.

overestimated at $\mathrm{C}$ - and $\mathrm{X}$-bands and the height IQR tends to increase with increasing frequency. This might depend on temporal decorrelation effects, which have been ignored in this simplified OVoG formulation and are likely more pronounced at higher frequencies. Any minor fluctuations of the height bias with the frequency (e.g., higher median in C-band than in $\mathrm{X}$-band for C3) are possibly due to the fact that the dualbaseline measurements employed for a specific crop at different frequencies do not necessarily share the same temporal baseline, even if they may have comparable $\kappa_{v}$ values.

For the remaining fields, one may observe that the dependence of the estimation error on the frequency is more pronounced. The height estimates of the wheat fields $\mathrm{W} 1$ and W4 and the barley field B3 are characterized by a reduced bias at X-band, whereas they undergo a considerable overestimation (e.g., height bias of $0.7 \mathrm{~m}$ for W1) at L-band. The retrieved height of the rape field $\mathrm{R}$, which is consistent with the ground measurements at L-band, is significantly underestimated at Cand X-bands (height bias of about $-0.55 \mathrm{~m}$, in the worst case).

One can identify three possible reasons to explain these discrepancies between the estimates and the measured values, as follows.

- The mismatch is due to the use of a restricted set of $\kappa_{v}$ values, which are typically higher for X-band than for L- and C-bands. As an example, the height overestimation 


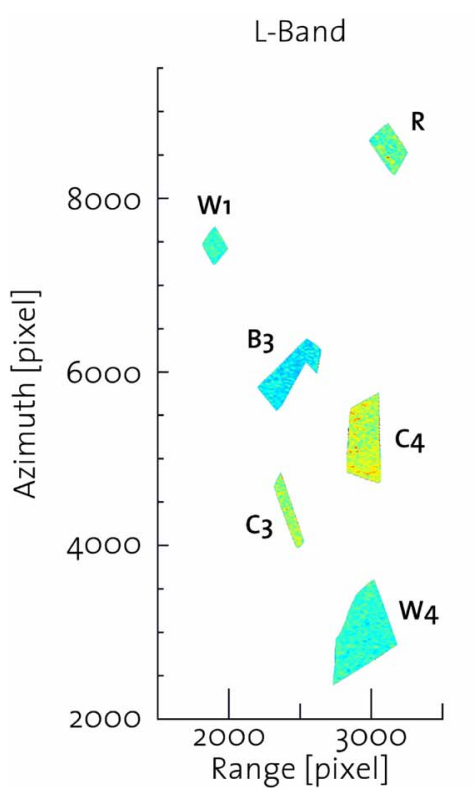

(a)

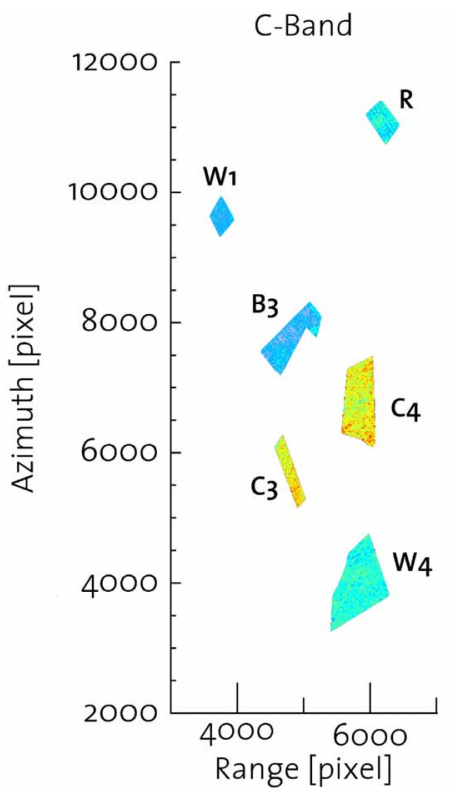

(b)

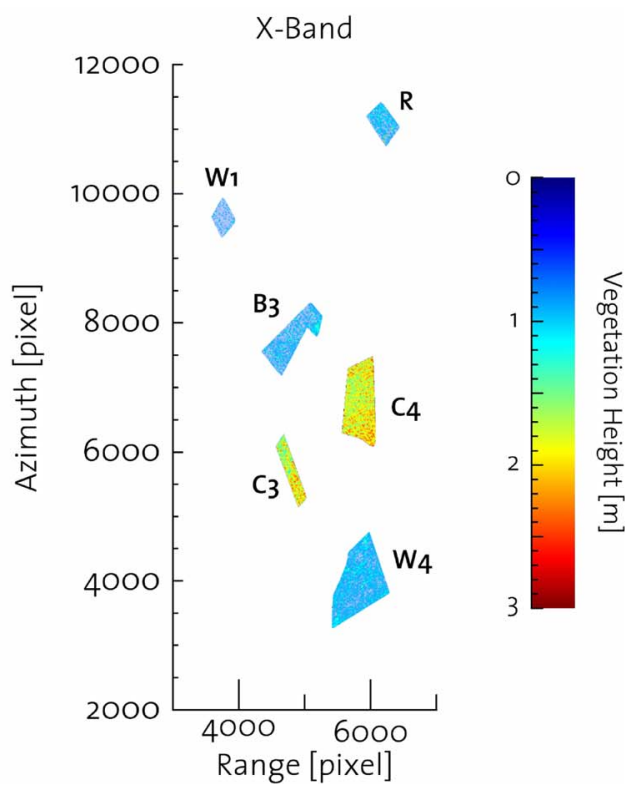

(c)

Fig. 25. Crop height estimation: estimated height at (a) L-band, (b) C-band, and (c) X-band.

at L- and C-bands for the wheat field W4 may be related to the small values of the available $\kappa_{z}$ and, hence, the poor sensitivity of the measured coherences to variations of the vegetation height. A pronounced height overestimation (up to $100 \%$ ) when $\kappa_{v}$ is very small was also found with a single-baseline approach [10] for wheat and barley at L-band. Interestingly, the height estimation error tends to decrease with increasing frequency also for $\mathrm{W} 1$ and B3. As for W4, this behavior could depend on the $\kappa_{v}$ values employed for the inversion, which are positively related to the sensor frequency.

- The measured observables are still affected by a residual geometric decorrelation (even after range spectral filtering) and other decorrelation sources: this can possibly justify the height overestimation at L-band, particularly for the wheat field $\mathrm{W} 1$ and the barley field B3, which are located in near range.

- The model either fails to properly describe the scattering scenario at a certain frequency or there is a lack of sensitivity of the observables to variations of the $\mathrm{OVoG}$ parameters (e.g., when the surface scattering power is dominant over volume scattering, or vice versa). For short dry plants such as in the barley field B3 [see Fig. 19(c)], it is reasonable to assume a strong scattering contribution from the underlying ground at low frequencies, such as L-band. This might result in a reduced sensitivity of the interferometric coherence to the vegetation height, making the estimation of such a parameter inaccurate. Note that the use of higher frequencies such as C- and $\mathrm{X}$-bands significantly improves the accuracy of the estimated height, as the wave interaction with the canopy likely increases and the relative contribution of the ground is reduced. On the other hand, agricultural crops with a dense canopy structure, such as the rape field $\mathrm{R}$ [see Fig. 19(f)] can be characterized, at high frequencies (e.g., $\mathrm{X}$-band), by a minor penetration depth and a negligible

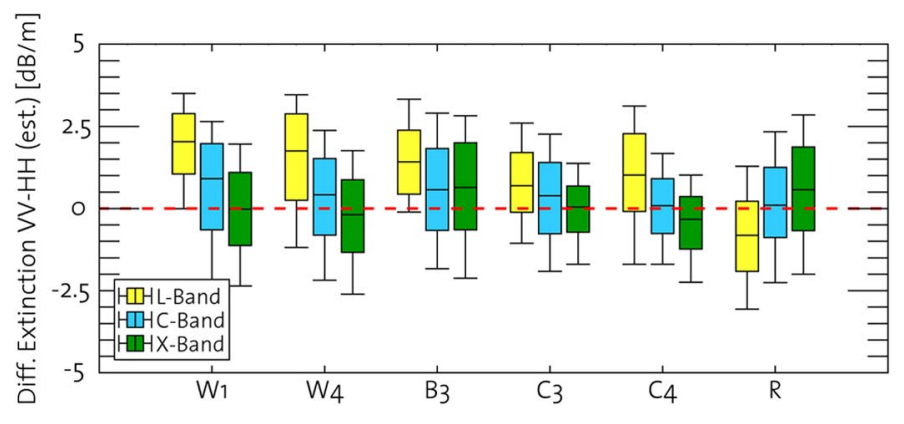

Fig. 26. Experimental data analysis: estimated differential extinction $\Delta \sigma=$ $\sigma_{\mathrm{VV}}-\sigma_{\mathrm{HH}}$ (dual-baseline case) at L-band (yellow), C-band (light blue), and $\mathrm{X}$-band (green).

ground-to-volume ratio, independent of polarization. In this case, the measured coherences become insensitive to the vegetation height, and the inversion results are inaccurate. We believe that this could explain the height underestimation at $\mathrm{C}$ - and $\mathrm{X}$-bands for the rape field. The consistency of the height estimates at L-band might be related to the fact that the extinction coefficients typically decrease, whereas the ground-to-volume ratios increase, with increasing frequencies.

Some early remarks can be pointed out from the estimated differential extinction $\Delta \sigma=\sigma_{\mathrm{VV}}-\sigma_{\mathrm{HH}}$ (see Fig. 26), through a comparison with previous studies [12], [13]. In particular, for the maize fields $\mathrm{C} 3$ and $\mathrm{C} 4, \Delta \sigma$ is consistently (75\% of the samples) greater than zero at L-band, and its median ranges between 0.7 and $1 \mathrm{~dB} / \mathrm{m}$. Such a positive offset (about $2 \mathrm{~dB} / \mathrm{m}$ for incidence angles greater than $38^{\circ}$ ) was previously found in [13]. In the same study, field measurements at C- and X-bands indicated no differential extinction, which is consistent with the results in Fig. 26. At low frequencies, one may also observe a good agreement between the estimates in Fig. 26 for the maize fields and those presented in [12]. Although characterized by a remarkable variability, the inversion results in [12] showed that 


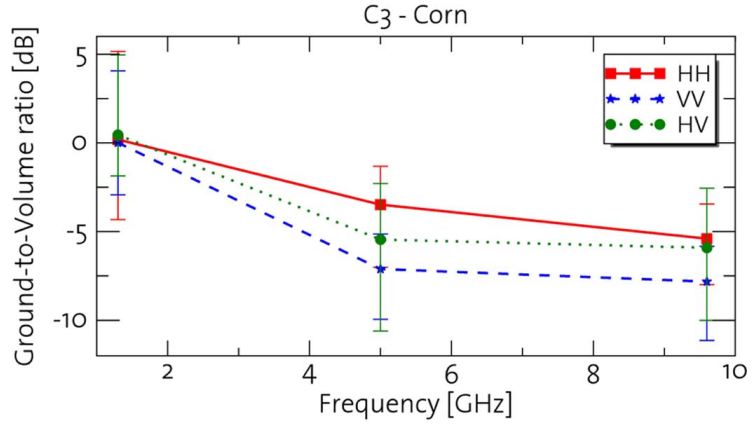

(a)

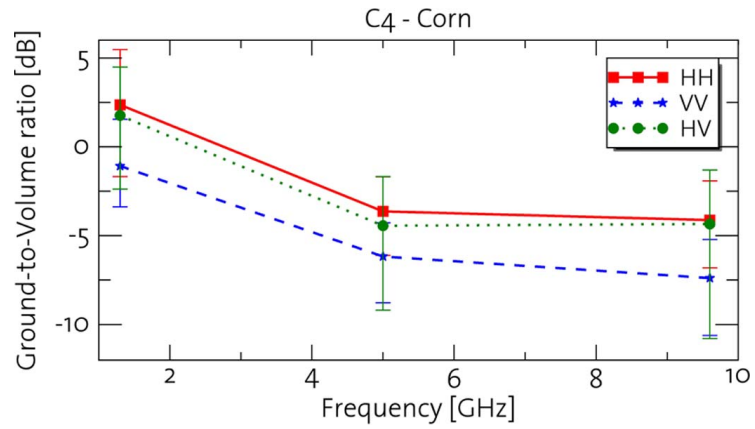

(b)

Fig. 27. Experimental data analysis: estimated ground-to-volume ratio $\mu$ as a function of the frequency for the (a) maize $\mathrm{C} 3$ and the (b) maize $\mathrm{C} 4$. The solid lines refer to the median, whereas the two whiskers represent the lower and upper quartiles.

the mean value of the retrieved VV extinction is higher than the $\mathrm{HH}$ extinction and their difference ranges between 0.5 and $1 \mathrm{~dB} / \mathrm{m}$, depending on the inversion strategy.

With regard to the rape field, a negative $\Delta \sigma$ is noticeable at L-band $(\Delta \sigma$ is lower than zero for almost $75 \%$ of the samples). Given the validity of the OVoG assumptions, this finding indicates that, while propagating through the canopy, the $H$-polarized wave undergoes a more severe attenuation than the $V$-polarized one. If the $H-V$ linear basis is the volume eigenpolarization basis, then it is reasonable to assume that the volume particles share a preferred horizontal orientation.

Although the $\mathrm{HH}$ and VV extinctions are different for maize and rape at L-band, previous studies [10], [12] on the same crop types provided robust estimates of the vegetation height (bias below 15\%) and the ground topography by using inversion approaches based on RVoG assumptions. The impact of the nonzero differential extinction on the accuracy of such estimates may depend on specific conditions (e.g., crop dielectric properties, $\kappa_{z}$, and sensor frequency), which deserve dedicated theoretical and experimental investigations.

\section{Dual-Baseline Case: Estimation of Ground-to-Volume Ratios}

The baseline configurations in Fig. 23 have been employed to estimate the ground-to-volume scattering ratios in the three polarimetric channels. Here, only the inversion results for the two maize fields are reported, as the estimated height for such fields is consistent with the in situ measurements at all frequencies (\%RMSD below 20\%).

The ground-to-volume ratios for the maize $\mathrm{C} 3$ and $\mathrm{C} 4$ are displayed as a function of the frequency in Fig. 27(a) and (b), respectively. In both cases, the median of $\mu_{\mathrm{VV}}$ is always lower than that of the other two channels, and the median of $\mu_{\mathrm{HH}}$ has the highest values. Furthermore, the median of all estimates decreases with the increasing frequency (at L-band, it ranges between -1 and $3 \mathrm{~dB}$; at $\mathrm{X}$-band, it ranges between -8 and $-5 \mathrm{~dB}$ ). Such a decreasing trend for maize is in line with expectations and consistent with previous studies [12]. At high frequencies (e.g., X-band), the vegetation canopy appears "denser," and hence, the scattering response from the ground is "weaker," as the incident wave interacts predominantly with leaf-scale objects.

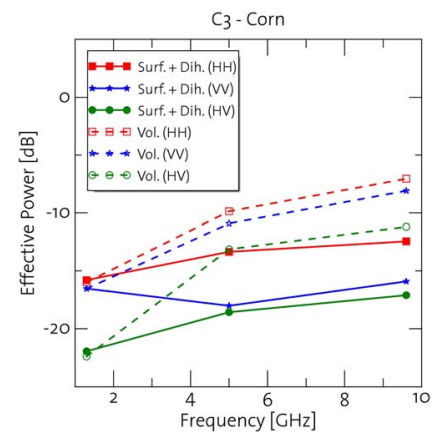

(a)

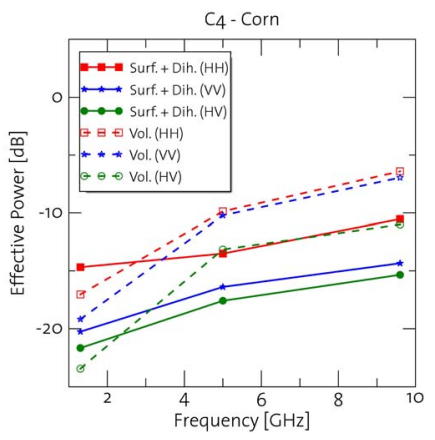

(b)
Fig. 28. Experimental data analysis: (solid lines) effective surface+dihedral power $\beta^{(S+D)}$ and (dashed lines) effective volume power $\beta^{(V)}$ as a function of the frequency for the (a) maize $\mathrm{C} 3$ and the (b) maize $\mathrm{C} 4$.

The estimated ground-to-volume ratios $\mu_{p q}((p, q)=$ $(H, H),(V, V),(H, V))$ in Fig. 27 can be used to compute the surface+dihedral effective power $\beta_{p q}^{(S+D)}$ and the volume effective power $\beta_{p q}^{(V)}$. From equations (7) and (8)

$$
\left\{\begin{array}{l}
\beta_{p q}^{0}=\beta_{p q}^{(S+D)}+\beta_{p q}^{(V)} \\
\mu_{p q}=\frac{\beta_{p q}^{(S+D)}}{\beta_{p q}^{(V)}}
\end{array}\right.
$$

where $\beta_{p q}^{0}=\vec{\omega}_{p q}^{\dagger}\left[T_{\mathrm{OVoG}}\right] \vec{\omega}_{p q}$ is the measured radar brightness.

The expressions in (28) are then combined to obtain $\beta_{p q}^{(S+D)}=\beta_{p q}^{0} \cdot \mu_{p q} /\left(1+\mu_{p q}\right)$ and $\beta_{p q}^{(V)}=\beta_{p q}^{0} /\left(1+\mu_{p q}\right)$.

The median of the estimated effective power (in $\mathrm{dB}$ ) is computed for the three polarimetric channels and reported in Fig. 28. These values tend to increase with the frequency, and the effective power in the $\mathrm{HV}$ channel is always lower than that in the $\mathrm{HH}$ and $\mathrm{VV}$ channels.

With regard to the maize field $\mathrm{C} 4$, the volume effective power $\beta_{\mathrm{VV}}^{(V)}<\beta_{\mathrm{HH}}^{(V)}$ at L-band $\left(\beta_{\mathrm{HH}}^{(V)}-\beta_{\mathrm{VV}}^{(V)}=2 \mathrm{~dB}\right)$, whereas no significant difference is found at $\mathrm{C}$ - and $\mathrm{X}$-bands. In this case, the estimated nonzero differential extinction at L-band (see Section V-C) may be a relevant factor for such a positive offset between $\beta_{\mathrm{HH}}^{(V)}$ and $\beta_{\mathrm{VV}}^{(V)}$ (i.e., $\beta_{\mathrm{VV}}^{(V)}$ undergoes a more severe reduction than $\beta_{\mathrm{HH}}^{(V)}$ because $\left.\sigma_{\mathrm{VV}}>\sigma_{\mathrm{HH}}\right)$.

The surface+dihedral effective power $\beta_{\mathrm{HH}}^{(S+D)}$ is significantly higher than $\beta_{\mathrm{VV}}^{(S+D)}$ at all frequencies, with $\beta_{\mathrm{HH}}^{(S+D)}-$ $\beta_{\mathrm{VV}}^{(S+D)}$ ranging between 3 and $5.5 \mathrm{~dB}$. Such a behavior may 
again be related to the nonzero differential extinction of the volume layer above the ground, but it may also be due to a strong dihedral ground-stalk scattering response.

\section{CONCLUSION}

This paper has presented a novel model-based inversion scheme, which takes advantage of the multibaseline extended observation space to estimate the whole set of the OVoG structural parameters. The inversion is achieved via the assumption that such unknowns are constant with respect to the vertical wavenumber $\kappa_{z}$ and that, for a fixed baseline, the ground phase does not change with the polarization channels.

The proposed algorithm has been initially assessed on a set of randomly generated $\mathrm{OVoG}$ scenarios and then applied to a collection of multi-baseline repeat-pass F-SAR data of the DLR, acquired over a rural area in Germany.

The best estimation performance is achieved for a subgroup of spatial baselines. When small baselines are employed, the lack of sensitivity of the Pol-InSAR coherence to the vegetation height results in a sizeable drop of the estimates accuracy if an additional decorrelation source is added. Furthermore, the phase ambiguities due to the excessive volume decorrelation degrades the algorithm performance when the height of ambiguity tends toward the actual vegetation height.

For a given set of baselines within the aforementioned subgroup, the sensitivity study over simulated scenarios has proved that the proposed algorithm is significantly stable over changes of the crop structure and robust against nonvolumetric decorrelation sources.

The preliminary assessment on the observed data showed clear dependence of the final estimates on the sensor frequency, as well as the existence of differential propagation effects within the crop canopy.

This study is intended as a first step toward the understanding of the potential of L-, C-, and X-bands in estimating the properties of different crops. It may also serve as a valuable support for evaluating the performance of current satellite systems (e.g., DLR's TanDEM-X [27]) and devising new acquisition strategies for Pol-InSAR crop parameter estimation (such as airborne campaigns or future satellite missions such as Tandem-L [28]).

In the future, the OVoG inversion scheme will be assessed over a collection of multitemporal Pol-InSAR data (DLR's F-SAR campaign, Wallerfing 2014), acquired over different crop types and throughout the whole plant growth cycle.

In addition, theoretical and experimental investigations will be conducted to ascertain the inversion performance of the proposed algorithm in comparison with the state-of-the-art singlebaseline inversion methods (e.g., three-stage inversion [3]).

\section{ACKNOWLEDGMENT}

The authors would like to thank the Microwaves and Radar Institute of the DLR for providing and processing the airborne data and the ground measurements used for this work. The data have been collected in the framework of the HGF-Alliance "Remote Sensing of Earth System Dynamics," HA-310.

\section{REFERENCES}

[1] S. R. Cloude and K. P. Papathanassiou, "Polarimetric SAR interferometry," IEEE Trans. Geosci. Remote Sens., vol. 36, no. 5, pp. 1551-1565, Sep. 1998.

[2] K. P. Papathanassiou and S. R. Cloude, "Single-baseline polarimetric SAR interferometry," IEEE Trans. Geosci. Remote Sens., vol. 39, no. 11, pp. 2352-2363, Nov. 2001.

[3] S. R. Cloude and K. P. Papathanassiou, "Three-stage inversion process for polarimetric SAR interferometry," Proc. Inst. Elect. Eng.-Radar Sonar Navig., vol. 150, no. 3, pp. 125-134, Jun. 2003.

[4] F. Garestier, P. C. Dubois-Fernandez, and I. Champion, "Forest height inversion using high-resolution P-band Pol-InSAR data," IEEE Trans. Geosci. Remote Sens., vol. 46, no. 11, pp. 3544-3559, Nov. 2008.

[5] I. Hajnsek, F. Kugler, S. K. Lee, and K. P. Papathanassiou, "Tropicalforest-parameter estimation by means of Pol-InSAR: The INDREX-II campaign," IEEE Trans. Geosci. Remote Sens., vol. 47, no. 2, pp. 481-493, Feb. 2009.

[6] F. Garestier, P. C. Dubois-Fernandez, and K. P. Papathanassiou, "Pine forest height inversion using single-pass X-Band PolInSAR data," IEEE Trans. Geosci. Remote Sens., vol. 46, no. 1, pp. 59-68, Jan. 2008.

[7] M. Neumann, L. Ferro-Famil, and A. Reigber, "Estimation of forest structure, ground, and canopy layer characteristics from multibaseline polarimetric interferometric SAR data," IEEE Trans. Geosci. Remote Sens., vol. 48, no. 3, pp. 1086-1104, Mar. 2010.

[8] I. Hajnsek and S. R. Cloude, "Pol-InSAR for agricultural vegetation parameter estimation," in Proc. IEEE IGARSS, Anchorage, AK, USA, 2004, pp. 1224-1227.

[9] J. M. Lopez-Sanchez, J. D. Ballester-Berman, and J. Fortuny-Guasch, "Indoor wide-band polarimetric measurements on maize plants: A study of the differential extinction coefficient," IEEE Trans. Geosci. Remote Sens., vol. 44, no. 4, pp. 758-767, Apr. 2006.

[10] J. M. Lopez-Sanchez, I. Hajnsek, and J. D. Ballester-Berman, "First demonstration of agriculture height retrieval with PolInSAR airborne data," IEEE Geosci. Remote Sens. Lett., vol. 9, no. 2, pp. 242-246, Mar. 2012.

[11] J. D. Ballester-Berman, J. M. Lopez-Sanchez, and J. Fortuny-Guasch, "Retrieval of biophysical parameters of agricultural crops using polarimetric SAR interferometry," IEEE Trans. Geosci. Remote Sens., vol. 43, no. 4, pp. 683-694, Apr. 2005.

[12] J. M. Lopez-Sanchez, J. D. Ballester-Berman, and Y. Marquez-Moreno, "Model limitations and parameter-estimation methods for agricultural applications of polarimetric SAR interferometry," IEEE Trans. Geosci. Remote Sens., vol. 45, no. 11, pp. 3481-3493, Nov. 2007.

[13] F. T. Ulaby, A. Tavakoli, and B. A. Thomas, "Microwave propagation constant for a vegetation canopy with vertical stalks," IEEE Trans. Geosci. Remote Sens., vol. GE-25, no. 6, pp. 714-725, Nov. 1987.

[14] R. N. Treuhaft and S. R. Cloude, "The structure of oriented vegetation from polarimetric interferometry," IEEE Trans. Geosci. Remote Sens., vol. 37, no. 5, pp. 2620-2624, Sep. 1999.

[15] R. N. Treuhaft and P. R. Siqueira, "Vertical structure of vegetated land surfaces from interferometric and polarimetric radar," Radio Sci., vol. 35, no. 1, pp. 141-177, Jan./Feb. 2000.

[16] S. R. Cloude, Polarisation: Applications in Remote Sensing. New York, NY, USA: Oxford Univ. Press, 2009.

[17] M. Lavalle, M. Simard, and S. Hensley, "A temporal decorrelation model for polarimetric radar interferometers," IEEE Trans. Geosci. Remote Sens., vol. 50, no. 7, pp. 2880-2888, Jul. 2012.

[18] I. Hajnsek, E. Pottier, and S. R. Cloude, "Inversion of surface parameters from polarimetric SAR," IEEE Trans. Geosci. Remote Sens., vol. 41, no. 4, pp. 727-744, Apr. 2003.

[19] M. Neumann, "Remote sensing of vegetation using multi-baseline polarimetric SAR interferometry: Theoretical modeling and physical parameter retrieval," Ph.D. dissertation, Institut d'électronique et de télécommunications de Rennes, Univ. Rennes 1, Rennes, France, 2009.

[20] S. R. Cloude, J. Fortuny, J. M. Lopez-Sanchez, and A. J. Sieber, "Wideband polarimetric radar inversion studies for vegetation layers," IEEE Trans. Geosci. Remote Sens., vol. 37, no. 5, pp. 2430-2441, Sep. 1999.

[21] M. Neumann et al., "Ground-agriculture separation by means of PolInSAR," in Proc. Polinsar, Frascati, Italy, 2007, pp. 1-7.

[22] J. D. Ballester-Berman and J. M. Lopez-Sanchez, "Coherence loci for a homogeneous volume over a double-bounce ground return," IEEE Geosci. Remote Sens. Lett., vol. 4, no. 2, pp. 317-321, Apr. 2007.

[23] J. D. Ballester-Berman and J. M. Lopez-Sanchez, "Combination of direct and double-bounce ground responses in the homogeneous oriented volume over ground model," IEEE Geosci. Remote Sens. Lett., vol. 8, no. 1, pp. 54-58, Jan. 2011. 
[24] M. Lavalle and K. Khun, "Three-baseline InSAR estimation of forest height," IEEE Geosci. Remote Sens. Lett., vol. 11, no. 10, pp. 1737-1741, Oct. 2014.

[25] L. Ferro-Famil, M. Neumann, and Y. Huang, "Multi-baseline PolInSAR statistical techniques for the characterization of distributed media," in Proc. IEEE IGARSS, Cape Town, South Africa, 2009, vol. 3, pp. III-971-III-974.

[26] F. Gatelli et al., "The wavenumber shift in SAR interferometry," IEEE Trans. Geosci. Remote Sens., vol. 32, no. 4, pp. 855-863, Jul. 1994.

[27] G. Krieger et al., "TanDEM-X: A satellite formation for high-resolution SAR interferometry," IEEE Trans. Geosci. Remote Sens., vol. 45, no. 11, pp. 3317-3341, Nov. 2007.

[28] I. Hajnsek et al., "Tandem-L: Science requirements and mission concept," in Proc. IEEE EUSAR, Berlin, Germany, Jun. 2014, pp. 1-4.

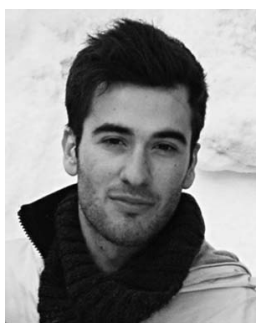

Manuele Pichierri received the B.Sc. degree in electronic and information engineering and the M.Sc. degree (cum laude) in electronic engineering from the University of Perugia, Perugia, Italy, in 2009 and 2011, respectively. His graduate studies thesis focused on the estimation of the urban heat island of Milan using spaceborne thermal-infrared sensors. He is currently working toward the Ph.D. degree in the Institute of Environmental Engineering, Swiss Federal Institute of Technology (ETH) Zurich, Zurich, Switzerland.

His research currently focuses on the characterization of the polarimetric interferometric scattering responses from agricultural crops and their dependence on frequency, vegetation moisture, and canopy structure.

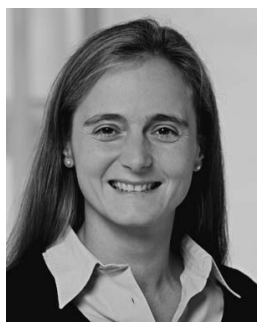

Irena Hajnsek (A'01-M'06-SM'09-F' 14) received the Dipl.(Hons.) degree from the Free University of Berlin, Berlin, Germany, in 1996 and the Dr.(Hons.) degree from the Friedrich Schiller University of Jena, Jena, Germany, in 2001.

Since November 2009, she has been a Professor of Earth observation with the Institute of Environmental Engineering, Swiss Federal Institute of Technology (ETH) Zurich, Zurich, Switzerland, and, at the same time, the Head of the polarimetric SAR interferometry research group with the Microwaves and Radar Institute, German Aerospace Center, Wessling, Germany. Her main research interests are in electromagnetic propagation and scattering theory, radar polarimetry, SAR and interferometric SAR data processing techniques, and environmental parameter modeling and estimation. Since 2010, she has been the Science Coordinator of the German satellite mission TanDEM-X.

Dr. Hajnsek has been a member of the IEEE Geoscience and Remote Sensing Society Administrative Committee since 2013. She was the Technical Program Cochair of the IEEE International Geoscience and Remote Sensing Symposium 2012 Symposium in Munich.

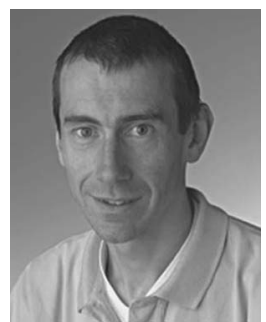

Konstantinos P. Papathanassiou (A'01-M'06SM'09-F'13) received the Dipl.Ing.(Hons.) and Dr.(Hons.) degrees from Graz University of Technology, Graz, Austria, in 1994 and 1999, respectively.

From 1992 to 1994 , he was with the Institute for Digital Image Processing (DIBAG), Joanneum Research, Graz. Between 1995 and 1999, he was with the Microwaves and Radar Institute, German Aerospace Center (DLR-HR), Wessling, Germany. From 1999 to 2000, he was a European Union Postdoctoral Fellow with Applied Electromagnetics, Fife, U.K. Since October 2000, he has been a Senior Scientist with DLR-HR, leading the Information Retrieval Research Group. He is a member of DLR's TanDEM-X and Tandem-L Science Teams, JAXA's ALOS-2 Cal-Val teams, the European Space Agency's BIOMASS mission Advisory Group, SAOCOMSC's Expert Team, JAXA's Kyoto and Carbon Initiative, and the National Aeronautics and Space Administration's Global Ecosystem Dynamics Investigation Lidar (GEDI) Mission Science Team. He has authored or coauthored over 100 publications in international journals, conferences, and workshops. His main research interests are in polarimetric and interferometric processing and calibration techniques, polarimetric SAR interferometry, and the quantitative parameter estimation from SAR data, as well as in SAR mission design and SAR mission performance analysis.

Dr. Papathanassiou was a recipient of the Symposium Prize Award of the IEEE Geoscience and Remote Sensing Society International Geoscience and Remote Sensing Symposium in 1998, the Best Paper Award of the European SAR Conference in 2002, the DLR Science Award in 2002, and the DLR Senior Scientist Award in 2011. 